\title{
Revised Michaelis-Menten Rate Law with Time-Varying Molecular Concentrations
}

\author{
Roktaek Lim ${ }^{1,7}$, Thomas L. P. Martin ${ }^{1,7}$, Junghun Chae ${ }^{2,7}$, Woojung Kim², Haneul Kim², \\ Cheol-Min Ghim ${ }^{2,3,}{ }^{*}$, and Pan-Jun Kim ${ }^{1,4,5,6, *}$ \\ ${ }^{1}$ Department of Biology, Hong Kong Baptist University, Kowloon, Hong Kong \\ ${ }^{2}$ Department of Physics, Ulsan National Institute of Science and Technology, Ulsan 44919, \\ Republic of Korea \\ ${ }^{3}$ Department of Biomedical Engineering, Ulsan National Institute of Science and Technology, \\ Ulsan 44919, Republic of Korea \\ ${ }^{4}$ Center for Quantitative Systems Biology \& Institute of Computational and Theoretical Studies, \\ Hong Kong Baptist University, Kowloon, Hong Kong \\ ${ }^{5}$ State Key Laboratory of Environmental and Biological Analysis, Hong Kong Baptist University, \\ Kowloon, Hong Kong \\ ${ }^{6}$ Abdus Salam International Centre for Theoretical Physics, 34151 Trieste, Italy \\ ${ }^{7}$ R.L., T.L.P.M., and J.C. contributed equally to this work. \\ *Author to whom correspondence should be addressed: C.-M.G. (email: cmghim@unist.ac.kr) \\ or P.-J.K. (email: panjunkim@hkbu.edu.hk)
}

\begin{abstract}
Despite over a century's use as a dominant paradigm in the description of biochemical rate processes, the Michaelis-Menten (MM) rate law stands on the restrictive assumption that the concentration of the complex of interacting molecules, at each moment, approaches an equilibrium much faster than the molecular concentration changes. The increasingly-appreciated, remedied form of the MM rate law is also based on this quasi-steady state assumption. Although this assumption may be valid for a range of biochemical systems, the exact extent of such systems is not clear. In this study, we relax the quasi-steady state requirement and propose the revised MM rate law for the interactions of molecules with active concentration changes over time. Our revised rate law, characterized by rigorously-derived time delay effects in molecular complex formation, improves the accuracy of models especially for proteinprotein and protein-DNA interactions. Our simulation and empirical data analysis show that the improvement is not limited to the quantitatively better characterization of the dynamics, but also allows the prediction for qualitatively new patterns in the systems of interest. The latter include the oscillation condition and period patterns of the mammalian circadian clock and the spontaneous rhythmicity in the degradation rates of circadian proteins, both not properly captured by the previous approaches. Moreover, our revised rate law is capable of more accurate parameter estimation. This work offers an analytical framework for understanding rich dynamics of biomolecular systems, which goes beyond the quasi-steady state assumption.
\end{abstract}




\section{INTRODUCTION}

Since proposed by Henri [1] and Michaelis and Menten [2], the Michaelis-Menten (MM) rate law has been the dominant framework for modeling the rates of enzyme-catalyzed reactions for over a century [1-4]. The MM rate law has also been widely adopted for describing other bimolecular interactions, such as reversible binding between proteins [5-7], between a gene and a transcription factor (TF) $[8,9]$, and between a receptor and a ligand $[10,11]$. The derivation of the MM rate law from the underlying biochemical mechanism is based on the steady-state approximation by Briggs and Haldane [3], referred to as the standard quasisteady state approximation (sQSSA) [12-14]. The SQSSA, however, is only valid when the enzyme concentration is low enough and thus the concentration of enzyme-substrate complex is negligible compared to substrate concentration [14]. This condition may be acceptable for many metabolic reactions with substrate concentrations that are typically far higher than the enzyme concentrations.

Nevertheless, in the case of protein-protein interactions in various cellular activities, the interacting proteins as the "enzymes" and "substrates" often show the concentrations comparable with each other [15-17]. Therefore, the use of the MM rate law for describing protein-protein interactions has been challenged in its rationale, with the modified alternative formula from the total quasi-steady state approximation (tQSSA) [12,13,18-24]. The tQSSAbased form is generally more accurate than the MM rate law from the SQSSA, for a broad range of combined molecular concentrations and thus for protein-protein interactions as well $[12,13,18-24]$. The superiority of the tQSSA has not only been proven in the quantitative, but also in the qualitative outcomes of systems, which the SQSSA sometimes fails to predict $[12,18]$. Later, we will provide the overview of the tQSSA and its relationship with the conventional MM rate law from the SQSSA.

Despite the correction of the MM rate law by the tQSSA, both the tQSSA and SQSSA still rely on the assumption that the concentration of the complex of interacting molecules, at each moment, approaches an equilibrium much faster than the molecular concentration changes $[12,14,21]$. Although this quasi-steady state assumption may work for a range of biochemical systems, the exact extent of such systems to follow that assumption is not clear. Numerous cellular processes do exhibit active molecular concentration changes over time, such as in circadian clock circuits or cell cycle systems $[6,7,18,25-28]$, calling for a better approach to even cover the time-varying molecular concentrations that may not strictly adhere to the quasi-steady state assumption.

In this study, we report the revision of the MM rate law, whereby the interaction of timevarying molecular components is more precisely described than by the existing approaches including the tQSSA. This revision is the correction of the TQSSA with rigorously-estimated time-delay effects, which improves the predictability for quantitative molecular-binding kinetics and retrieves the tQSSA in steady conditions. Our formulation well accounts for the nontrivial oscillatory dynamics and empirical patterns of biomolecular systems with the relevant analytical insights, which are not captured by the previous methods-the tQSSA and SQSSA. This work offers a new mathematical framework for interrogating rich dynamics of molecular interactions in various biochemical contexts.

\section{THEORY OVERVIEW AND DERIVATION}

\section{A. General formulation}

We consider two different molecules $A$ and $B$ that bind to each other and form complex $A B$. For example, $A$ and $B$ may represent two participant proteins in heterodimer formation, a chemical substrate and an enzyme in a metabolic reaction, and a solute and a transporter in 
membrane transport. The concentration of the complex $\mathrm{AB}$ at time $t$, denoted by $C(t)$, changes over time as in the following equation:

$$
\frac{\mathrm{d} C(t)}{\mathrm{d} t}=k_{\mathrm{a}}[A(t)-C(t)][B(t)-C(t)]-k_{\delta} C(t) .
$$

Here, $A(t)$ and $B(t)$ denote the total concentrations of $\mathrm{A}$ and $\mathrm{B}$, respectively, and hence $A(t)-C(t)$ and $B(t)-C(t)$ correspond to the concentrations of free $\mathrm{A}$ and $\mathrm{B}$. $k_{\mathrm{a}}$ denotes the association rate of free $\mathrm{A}$ and B. $k_{\delta}$ is defined as $k_{\delta} \equiv k_{\mathrm{d}}+r_{\mathrm{c}}+k_{\text {loc }}+k_{\mathrm{dlt}}$ where $k_{\mathrm{d}}$, $k_{\mathrm{loc}}$, and $k_{\mathrm{dlt}}$ stand for the dissociation, translocation, and dilution rates of $\mathrm{AB}$, respectively, and $r_{\mathrm{c}}$ for the chemical conversion or translocation rate of $\mathrm{A}$ or $\mathrm{B}$ upon the formation of $\mathrm{AB}$.

Using the notations $\tau \equiv k_{\delta} t, K \equiv k_{\delta} / k_{\mathrm{a}}, \bar{A}(\tau) \equiv A(t) / K, \bar{B}(\tau) \equiv B(t) / K$, and $\bar{C}(\tau) \equiv$ $C(t) / K$, one can rewrite Eq. (1) as

$$
\frac{\mathrm{d} \bar{C}(\tau)}{\mathrm{d} \tau}=[\bar{A}(\tau)-\bar{C}(\tau)][\bar{B}(\tau)-\bar{C}(\tau)]-\bar{C}(\tau) .
$$

By definition, $C(t) \leq A(t)$ and $C(t) \leq B(t)$, and therefore

$$
C(t) \leq \min [A(t), B(t)] \text { i.e., } \bar{C}(\tau) \leq \min [\bar{A}(\tau), \bar{B}(\tau)]\} \text {. }
$$

On the other hand, Eq. (2) is equivalent to

$$
\frac{\mathrm{d} \bar{C}(\tau)}{\mathrm{d} \tau}=\left[\bar{C}(\tau)-\bar{C}_{\mathrm{tQ}}(\tau)\right]\left\{\bar{C}(\tau)-\left[\bar{C}_{\mathrm{tQ}}(\tau)+\sqrt{\Delta_{\mathrm{tQ}}(\tau)}\right]\right\}
$$

where $\bar{C}_{\mathrm{tQ}}(\tau)$ and $\Delta_{\mathrm{tQ}}(\tau)$ are given by

$$
\begin{aligned}
\bar{C}_{\mathrm{tQ}}(\tau) & \equiv \frac{1}{2}\left[1+\bar{A}(\tau)+\bar{B}(\tau)-\sqrt{\Delta_{\mathrm{tQ}}(\tau)}\right] \\
\Delta_{\mathrm{tQ}}(\tau) \equiv & {[1+\bar{A}(\tau)+\bar{B}(\tau)]^{2}-4 \bar{A}(\tau) \bar{B}(\tau) } \\
& =1+2[\bar{A}(\tau)+\bar{B}(\tau)]+[\bar{A}(\tau)-\bar{B}(\tau)]^{2} .
\end{aligned}
$$

In the tQSSA, the assumption is that $C(t)$ approaches the equilibrium fast enough each time, given the values of $A(t)$ and $B(t)[12,21]$. To understand this idea, notice that $\bar{C}^{\prime}(\tau) \rightarrow 0$ in Eq. (4) when $\bar{C}(\tau) \rightarrow \bar{C}_{\mathrm{tQ}}(\tau)$ given the values of $\bar{A}(\tau)$ and $\bar{B}(\tau)$ [we use symbol' for a derivative, such as $\bar{C}^{\prime}(\tau)$ here]. One can prove that $\bar{C}_{\mathrm{tQ}}(\tau) \leq \min [\bar{A}(\tau), \bar{B}(\tau)]$ and thus Eq. (3) is naturally satisfied when $\bar{C}(\tau)=\bar{C}_{\mathrm{tQ}}(\tau)$. The other nominal solution of $\bar{C}^{\prime}(\tau)=0$ in Eq. (4) does not satisfy Eq. (3) and is thus physically senseless.

According to the tQSSA, one takes an estimate $\bar{C}(\tau) \approx \bar{C}_{\mathrm{tQ}}(\tau)$, or equivalently, $C(t) \approx$ $C_{\mathrm{tQ}}(t)$ with this form:

$$
C_{\mathrm{tQ}}(t) \equiv \frac{1}{2}\left\{K+A(t)+B(t)-\sqrt{[K+A(t)+B(t)]^{2}-4 A(t) B(t)}\right\} .
$$

As mentioned earlier, the tQSSA is generally more accurate than the conventional MM rate law $[12,13,18-24]$. To obtain the MM rate law, consider a rather specific condition,

$$
B(t) \ll K+A(t) \text { or } A(t) \ll K+B(t) \text { [i.e., } \bar{B}(\tau) \ll 1+\bar{A}(\tau) \text { or } \bar{A}(\tau) \ll 1+\bar{B}(\tau) \text { ]. (8) }
$$

In this condition, the Padé approximant for $C_{\mathrm{tQ}}(t)$ takes the following form:

$$
C_{\mathrm{tQ}}(t) \approx \frac{A(t) B(t)}{K+A(t)+B(t)}\left[\text { i.e., } \bar{C}_{\mathrm{tQ}}(\tau) \approx \frac{\bar{A}(\tau) \bar{B}(\tau)}{1+\bar{A}(\tau)+\bar{B}(\tau)}\right] .
$$

Considering Eq. (9), Eq. (8) is similar to the condition $C_{\mathrm{tQ}}(t) / A(t) \ll 1$ or $C_{\mathrm{tQ}}(t) / B(t) \ll 1$. In other words, Eq. (9) would be valid when the concentration of $A B$ complex is negligible compared to either A or B's concentration. This condition is essentially identical to the assumption in the SQSSA resulting in the MM rate law [14]. In the example of a typical metabolic reaction with $B(t) \ll A(t)$ for substrate $\mathrm{A}$ and enzyme $\mathrm{B}$, Eq. (8) is automatically satisfied and Eq. (9) further reduces to the familiar $\mathrm{MM}$ rate law $C_{\mathrm{tQ}}(t) \approx A(t) B(t) /[K+$ $A(t)]$, the same outcome of the sQSSA [1-4,12-14]. Clearly, $K$ here is the Michaelis constant, commonly known as $K_{\mathrm{M}}$.

The application of the MM rate law beyond the condition in Eq. (8) invites a risk of erroneous modeling results, whereas the tQSSA is relatively free of such errors and has wider applicability $[12,13,18-24]$. Still, both the tQSSA and SQSSA stand on the quasi-steady state assumption that $C(t)$ approaches the equilibrium fast enough each time before the marked temporal change of $A(t)$ or $B(t)$. Next, we will relieve this assumption and improve the approximation of $C(t)$ in the case of time-varying $A(t)$ and $B(t)$, as the main objective of this study. 
Suppose that $C(t)$ may not necessarily approach the equilibrium $C_{\mathrm{tQ}}(t)$ but stays within some distance from it, satisfying the following relation:

$$
\left|\bar{C}(\tau)-\bar{C}_{\mathrm{tQ}}(\tau)\right| \ll \sqrt{\Delta_{\mathrm{tQ}}(\tau)} .
$$

We will later show that this relation is readily satisfied in physiologically-relevant conditions. This relation allows us to discard $\left[\bar{C}(\tau)-\bar{C}_{\mathrm{tQ}}(\tau)\right]^{2}$ compared to $\sqrt{\Delta_{\mathrm{tQ}}(\tau)}\left[\bar{C}(\tau)-\bar{C}_{\mathrm{tQ}}(\tau)\right]$ and thereby reduce Eq. (4) to

$$
\frac{\mathrm{d} \bar{C}(\tau)}{\mathrm{d} \tau} \approx \sqrt{\Delta_{\mathrm{tQ}}(\tau)}\left[\bar{C}_{\mathrm{tQ}}(\tau)-\bar{C}(\tau)\right]
$$

The solution of Eq. (11) is given by

$$
\bar{C}(\tau) \approx \int_{\tau_{0}}^{\tau} \sqrt{\Delta_{\mathrm{tQ}}\left(\tau^{\prime}\right)} \bar{C}_{\mathrm{tQ}}\left(\tau^{\prime}\right) e^{-\int_{\tau^{\prime}}^{\tau} \sqrt{\Delta_{\mathrm{tQ}}\left(\tau^{\prime \prime}\right)} \mathrm{d} \tau^{\prime \prime}} \mathrm{d} \tau^{\prime}+\bar{C}\left(\tau_{0}\right) e^{-\int_{\tau_{0}}^{\tau} \sqrt{\Delta_{\mathrm{tQ}}\left(\tau^{\prime}\right)} \mathrm{d} \tau^{\prime}},
$$

where $\tau_{0}$ denotes an arbitrarily assigned, initial point of $\tau$. Assume that $\sqrt{\Delta_{\mathrm{tQ}}\left(\tau^{\prime}\right)}$ changes rather slowly over $\tau^{\prime}$ to satisfy

$$
\sqrt{\Delta_{\mathrm{tQ}}\left(\tau^{\prime}\right)} \approx \sqrt{\Delta_{\mathrm{tQ}}(\tau)} \text { for } \tau^{\prime} \text { in the range } \tau-\frac{1}{\sqrt{\Delta_{\mathrm{tQ}}(\tau)}} \lesssim \tau^{\prime} \leq \tau .
$$

As we will show later, Eq. (13) is satisfied as readily as Eq. (10) in physiologically-relevant conditions. With Eq. (13), Eq. (12) for $\tau \gg \tau_{0}+\Delta_{\mathrm{tQ}}^{-1 / 2}\left(\tau_{0}\right)$ is further approximated as

$$
\bar{C}(\tau) \approx \sqrt{\Delta_{\mathrm{tQ}}(\tau)} \int_{-\infty}^{\tau} \bar{C}_{\mathrm{tQ}}\left(\tau^{\prime}\right) e^{-\sqrt{\Delta_{\mathrm{tQ}}(\tau)}\left(\tau-\tau^{\prime}\right)} \mathrm{d} \tau^{\prime} .
$$

The Taylor expansion $\bar{C}_{\mathrm{tQ}}\left(\tau^{\prime}\right)=\bar{C}_{\mathrm{tQ}}(\tau)-\left(\tau-\tau^{\prime}\right) \bar{C}_{\mathrm{tQ}}^{\prime}(\tau)+\left(\tau-\tau^{\prime}\right)^{2} \bar{C}_{\mathrm{tQ}}^{\prime \prime}(\tau) / 2-\cdots$ leads Eq. (14) to

$$
\begin{aligned}
\bar{C}(\tau) & \approx \bar{C}_{\mathrm{tQ}}(\tau)-\frac{1}{\sqrt{\Delta_{\mathrm{tQ}}(\tau)}} \frac{\mathrm{d} \bar{C}_{\mathrm{tQ}}(\tau)}{\mathrm{d} \tau} \int_{0}^{\infty} x e^{-x} \mathrm{~d} x+\frac{1}{2 \Delta_{\mathrm{tQ}}(\tau)} \frac{\mathrm{d}^{2} \bar{C}_{\mathrm{tQ}}(\tau)}{\mathrm{d} \tau^{2}} \int_{0}^{\infty} x^{2} e^{-x} \mathrm{~d} x-\cdots \\
& =\bar{C}_{\mathrm{tQ}}(\tau)-\frac{1}{\sqrt{\Delta_{\mathrm{tQ}}(\tau)}} \frac{\mathrm{d} \bar{C}_{\mathrm{tQ}}(\tau)}{\mathrm{d} \tau}+\frac{1}{\Delta_{\mathrm{tQ}}(\tau)} \frac{\mathrm{d}^{2} \bar{C}_{\mathrm{tQ}}(\tau)}{\mathrm{d} \tau^{2}}-\cdots \\
& =\bar{C}_{\mathrm{tQe}}(\tau)+\frac{1}{\Delta_{\mathrm{tQ}}(\tau)} \frac{\mathrm{d}^{2} \overline{\mathrm{C}}_{\mathrm{tQ}}(\tau)}{\mathrm{d} \tau^{2}}-\cdots
\end{aligned}
$$

where $\bar{C}_{\mathrm{tQe}}(\tau)$ is defined as

$$
\bar{C}_{\mathrm{tQe}}(\tau) \equiv \bar{C}_{\mathrm{tQ}}(\tau)-\frac{1}{\sqrt{\Delta_{\mathrm{tQ}}(\tau)}} \frac{\mathrm{d} \overline{\mathrm{tQ}}_{\mathrm{Q}}(\tau)}{\mathrm{d} \tau}
$$

For the approximation of $\bar{C}(\tau)$, one may be tempted to use $\bar{C}_{\mathrm{tQe}}(\tau)$ in Eq. (16). However, as proven in Text S1, the sheer use of $\bar{C}_{\mathrm{tQe}}(\tau)$ is susceptible to the overestimation of the amplitude of $\bar{C}(\tau)$ when $\bar{C}(\tau)$ is rhythmic over time. To detour this overestimation problem, we take the Taylor expansion of the time-delayed form of $\bar{C}_{\mathrm{tQ}}(\tau)$ :

$$
\bar{C}_{\mathrm{tQ}}\left[\tau-\frac{1}{\sqrt{\Delta_{\mathrm{tQ}}(\tau)}}\right]=\bar{C}_{\mathrm{tQ}}(\tau)-\frac{1}{\sqrt{\Delta_{\mathrm{tQ}}(\tau)}} \frac{\mathrm{d} \bar{C}_{\mathrm{tQ}}(\tau)}{\mathrm{d} \tau}+\frac{1}{2 \Delta_{\mathrm{tQ}}(\tau)} \frac{\mathrm{d}^{2} \overline{\mathrm{C}}_{\mathrm{QQ}}(\tau)}{\mathrm{d} \tau^{2}}-\cdots
$$

Strikingly, the zeroth-order and first-order derivative terms of $\bar{C}_{\mathrm{tQ}}(\tau)$ on the right-hand side above are identical to $\bar{C}_{\text {tQe }}(\tau)$, and the second-order derivative term still covers a half of that term in Eq. (15). Hence, $\bar{C}_{\mathrm{tQ}}\left[\tau-\Delta_{\mathrm{tQ}}^{-1 / 2}(\tau)\right]$ and correspondingly, $C_{\mathrm{tQ}}\left[t-k_{\delta}^{-1} \Delta_{\mathrm{tQ}}^{-1 / 2}(t)\right]$ bear the potential for the approximants of $\bar{C}(\tau)$ and $C(t)$, respectively. Besides, the overestimation of the amplitude of rhythmic $\bar{C}(\tau)$ by $\bar{C}_{\mathrm{tQ}}\left[\tau-\Delta_{\mathrm{tQ}}^{-1 / 2}(\tau)\right]$ would not be as serious as by $\bar{C}_{\mathrm{tQe}}(\tau)$ and at worst equals that by $\bar{C}_{\mathrm{tQ}}(\tau)$ (Text S1), because $\bar{C}_{\mathrm{tQ}}\left[\tau-\Delta_{\mathrm{tQ}}^{-1 / 2}(\tau)\right]$ and $\bar{C}_{\mathrm{tQ}}(\tau)$ themselves have the same amplitudes.

The distinct feature of $\bar{C}_{\mathrm{tQ}}\left[\tau-\Delta_{\mathrm{tQ}}^{-1 / 2}(\tau)\right]$ or equivalent $C_{\mathrm{tQ}}\left[t-k_{\delta}^{-1} \Delta_{\mathrm{tQ}}^{-1 / 2}(t)\right]$ is the inclusion of an effective time delay $k_{\delta}^{-1} \Delta_{\mathrm{tQ}}^{-1 / 2}(t)$ in the formation of $\mathrm{AB}$ complex. As we will address later, this feature helps to capture intriguing biomolecular dynamics with time-varying 
molecular concentrations, which is not covered by the tQSSA. The effective time delay $k_{\delta}^{-1} \Delta_{\mathrm{tQ}}^{-1 / 2}(t)$ becomes prominent for small $k_{\delta}$ and for unsaturated molecules $\mathrm{A}$ and $\mathrm{B}$ (in their binding) with comparable concentrations-with small $\bar{A}(\tau)+\bar{B}(\tau)$ and $[\bar{A}(\tau)-\bar{B}(\tau)]^{2}$ in Eq. (6). In other words, in the opposite limit, the time delay becomes short and then $\bar{C}_{\mathrm{tQ}}\left[\tau-\Delta_{\mathrm{tQ}}^{-1 / 2}(\tau)\right]$ converges to $\bar{C}_{\mathrm{tQ}}(\tau)$, the tQSSA.

One caveat with the use of $\bar{C}_{\mathrm{tQ}}\left[\tau-\Delta_{\mathrm{tQ}}^{-1 / 2}(\tau)\right]$ to estimate $\bar{C}(\tau)$ is that $\bar{C}_{\mathrm{tQ}}\left[\tau-\Delta_{\mathrm{tQ}}^{-1 / 2}(\tau)\right]$ may not necessarily satisfy the relation $\bar{C}_{\mathrm{tQ}}\left[\tau-\Delta_{\mathrm{tQ}}^{-1 / 2}(\tau)\right] \leq \min [\bar{A}(\tau), \bar{B}(\tau)]$ favored by Eq. (3). As a practical safeguard to avoid this problem, we propose the following approximant for $\bar{C}(\tau)$ consistent with Eq. (3):

$$
\bar{C}_{\gamma}(\tau) \equiv \min \left\{\bar{C}_{\mathrm{tQ}}\left[\tau-\Delta_{\mathrm{tQ}}^{-1 / 2}(\tau)\right], \bar{A}(\tau), \bar{B}(\tau)\right\}
$$

The corresponding approximant for $C(t)$ is

$$
C_{\gamma}(t) \equiv \min \left\{C_{\mathrm{tQ}}\left[t-k_{\delta}^{-1} \Delta_{\mathrm{tQ}}^{-1 / 2}(t)\right], A(t), B(t)\right\} .
$$

As will be demonstrated later, the accuracy of this approximation $\left[\bar{C}_{\gamma}(\tau)\right.$ or $\left.C_{\gamma}(t)\right]$ surpasses that of the tQSSA $\left[\bar{C}_{\mathrm{tQ}}(\tau)\right.$ or $\left.C_{\mathrm{tQ}}(t)\right]$. In the case where Eq. (8) is satisfied, $\bar{C}_{\mathrm{tQ}}\left[\tau-\Delta_{\mathrm{tQ}}^{-1 / 2}(\tau)\right]$ and $C_{\mathrm{tQ}}\left[t-k_{\delta}^{-1} \Delta_{\mathrm{tQ}}^{-1 / 2}(t)\right]$ in Eqs. (18) and (19) can be further approximated by the MM-like forms in Eq. (9) with the time-delay terms $\Delta_{\mathrm{tQ}}^{-1 / 2}(\tau) \approx 1 /[1+\bar{A}(\tau)+\bar{B}(\tau)]$ and $k_{\delta}^{-1} \Delta_{\mathrm{tQ}}^{-1 / 2}(t) \approx k_{\delta}^{-1} K /[K+A(t)+B(t)]$. On the other hand, Eqs. (18) and (19) are ill-defined for $\tau-\Delta_{\mathrm{tQ}}^{-1 / 2}(\tau)<\tau_{0}$ where $\tau_{0}$ is an initial point of $\tau$. In fact, from the last term in Eq. (12), $\tau$ should satisfy $\tau \gg \tau_{0}+\Delta_{\mathrm{tQ}}^{-1 / 2}\left(\tau_{0}\right)$ for the application of any rate law [e.g., Eq. (18) or (19), the tQSSA, and the SQSSA] whose form does not depend on the initial conditions.

One may question the analytical utility of $\bar{C}_{\gamma}(\tau)$ and $C_{\gamma}(t)$ in Eqs. (18) and (19), regarding the apparent complexity of their mathematical structure. In Sec. III.E, our approach will be used to deliver valuable analytical insights into the system whose dynamics is otherwise not interpretable by the tQSSA or SQSSA.

To clarify the preconditions for the use of our approximation, we first revisit the condition in Eq. (10). Replacing $\bar{C}(\tau)$ in Eq. (10) by $\bar{C}_{\text {tQe }}(\tau)$ in Eqs. (15) and (16) leads to the following selfconsistency condition:

where $\bar{C}_{\mathrm{tQ}}^{\prime}(\tau)$ is given by

$$
\varepsilon_{1}(\tau) \equiv \frac{1}{\Delta_{\mathrm{tQ}}(\tau)}\left|\frac{\mathrm{d} \bar{c}_{\mathrm{tQ}}(\tau)}{\mathrm{d} \tau}\right| \ll 1,
$$

$$
\frac{\mathrm{d} \bar{C}_{\mathrm{tQ}}(\tau)}{\mathrm{d} \tau}=\frac{1}{\sqrt{\Delta_{\mathrm{tQ}}(\tau)}}\left\{\left[\bar{B}(\tau)-\bar{C}_{\mathrm{tQ}}(\tau)\right] \bar{A}(\tau) \mu_{\mathrm{A}}(\tau)+\left[\bar{A}(\tau)-\bar{C}_{\mathrm{tQ}}(\tau)\right] \bar{B}(\tau) \mu_{\mathrm{B}}(\tau)\right\}
$$

Here, $\mu_{\mathrm{A}}(\tau) \equiv \bar{A}^{\prime}(\tau) / \bar{A}(\tau), \mu_{\mathrm{B}}(\tau) \equiv \bar{B}^{\prime}(\tau) / \bar{B}(\tau)$, and the derivation of Eq. (21) is straightforward from Eq. (5). For the sake of simplicity, we will keep using these notations $\mu_{\mathrm{A}}(\tau)$ and $\mu_{\mathrm{B}}(\tau)$ throughout this work. Next, we revisit another condition in Eq. (13). By applying $\sqrt{\Delta_{\mathrm{tQ}}\left(\tau^{\prime}\right)} \approx \sqrt{\Delta_{\mathrm{tQ}}(\tau)}+\left(\tau^{\prime}-\tau\right)\left[\sqrt{\Delta_{\mathrm{tQ}}(\tau)}\right]^{\prime}$ to Eq. (13), we obtain $\Delta_{\mathrm{tQ}}^{-1}(\tau)\left[\sqrt{\Delta_{\mathrm{tQ}}(\tau)}\right]^{\prime} \ll 1$. By the definition of $\Delta_{\mathrm{tQ}}(\tau)$ in Eq. (6), this condition is equivalent to

$$
\varepsilon_{2}(\tau) \equiv \Delta_{\mathrm{tQ}}^{-\frac{3}{2}}(\tau)\left|[1+\bar{A}(\tau)-\bar{B}(\tau)] \bar{A}(\tau) \mu_{\mathrm{A}}(\tau)+[1+\bar{B}(\tau)-\bar{A}(\tau)] \bar{B}(\tau) \mu_{\mathrm{B}}(\tau)\right| \ll 1 .
$$

The last condition below arises from the comparison between Eqs. (15) and (17), which show the difference of $\sim \Delta_{\mathrm{tQ}}^{-1}(\tau) \bar{C}_{\mathrm{tQ}}^{\prime \prime}(\tau) / 2$ :

$$
\varepsilon_{\gamma}(\tau) \equiv \frac{1}{2 \Delta_{\mathrm{tQ}}(\tau) \bar{C}_{\mathrm{tQ}}\left[\tau-\Delta_{\mathrm{tQ}}^{-1 / 2}(\tau)\right]}\left|\frac{\mathrm{d}^{2} \bar{C}_{\mathrm{tQ}}(\tau)}{\mathrm{d} \tau^{2}}\right| \ll 1,
$$

where $\bar{C}_{\mathrm{tQ}}^{\prime \prime}(\tau)$ is obtained from Eq. (21) as 


$$
\begin{gathered}
\frac{\mathrm{d}^{2} \bar{C}_{\mathrm{tQ}}(\tau)}{\mathrm{d} \tau^{2}}=\Delta_{\mathrm{tQ}}^{-\frac{3}{2}}(\tau)\left\{\left\{\Delta_{\mathrm{tQ}}(\tau)-2 \bar{A}(\tau)\left[1+\bar{A}(\tau)-\bar{C}_{\mathrm{tQ}}(\tau)\right]\right\}\left[\bar{B}(\tau)-\bar{C}_{\mathrm{tQ}}(\tau)\right] \bar{A}(\tau) \mu_{\mathrm{A}}^{2}(\tau)+\right. \\
\left\{\Delta_{\mathrm{tQ}}(\tau)-2 \bar{B}(\tau)\left[1+\bar{B}(\tau)-\bar{C}_{\mathrm{tQ}}(\tau)\right]\right\}\left[\bar{A}(\tau)-\bar{C}_{\mathrm{tQ}}(\tau)\right] \bar{B}(\tau) \mu_{\mathrm{B}}^{2}(\tau)+\left\{1+\Delta_{\mathrm{tQ}}(\tau)-\right. \\
\left.[\bar{A}(\tau)-\bar{B}(\tau)]^{2}\right\} \bar{A}(\tau) \bar{B}(\tau) \mu_{\mathrm{A}}(\tau) \mu_{\mathrm{B}}(\tau)+\Delta_{\mathrm{tQ}}(\tau)\left\{\left[\bar{B}(\tau)-\bar{C}_{\mathrm{tQ}}(\tau)\right] \bar{A}(\tau) \frac{\mathrm{d} \mu_{\mathrm{A}}(\tau)}{\mathrm{d} \tau}+\right. \\
\left.\left.\left[\bar{A}(\tau)-\bar{C}_{\mathrm{tQ}}(\tau)\right] \bar{B}(\tau) \frac{\mathrm{d} \mu_{\mathrm{B}}(\tau)}{\mathrm{d} \tau}\right\}\right\} .
\end{gathered}
$$

In summary, our approximant $\bar{C}_{\gamma}(\tau)$ shall work when Eqs. (20), (22), and (23) are satisfied. As we will show later, Eqs. (20) and (22) are in fact easy to satisfy and thus only Eq. (23) tends to serve as the relevant factor of the validity of $\bar{C}_{\gamma}(\tau)$.

On the other hand, the tQSSA would be valid in the following condition from Eq. (15), instead of the condition in Eq. (23):

$$
\varepsilon_{\mathrm{tQ}}(\tau) \equiv \frac{1}{\bar{C}_{\mathrm{tQ}}(\tau)}\left|\frac{1}{\sqrt{\Delta_{\mathrm{tQ}}(\tau)}} \frac{\mathrm{d} \bar{C}_{\mathrm{tQ}}(\tau)}{\mathrm{d} \tau}-\frac{1}{\Delta_{\mathrm{tQ}}(\tau)} \frac{\mathrm{d}^{2} \bar{C}_{\mathrm{tQ}}(\tau)}{\mathrm{d} \tau^{2}}\right| \ll 1,
$$

where $\bar{C}_{\mathrm{tQ}}^{\prime}(\tau)$ and $\bar{C}_{\mathrm{tQ}}^{\prime \prime}(\tau)$ are given by Eqs. (21) and (24), respectively. Note that this condition for the tQSSA is more rigorous than the previously-reported condition [21].

\section{B. Transcription factor-DNA binding case}

Thus far, we have implicitly assumed the continuous nature of molecular concentrations as in Eq. (1) with the time derivative of $C(t)$. However, there exist biomolecular events that fundamentally deviate from this assumption. For example, a transcription factor (TF) binds to a DNA molecule in the nucleus to regulate mRNA expression and the number of such a TFDNA assembly would be either 1 or 0 for a particular DNA site, which can afford at most one copy of the TF. This inherently discrete nature of the TF-DNA binding number is seemingly contrasted with the continuity of the molecular complex level in Eq. (1). To rigorously describe this TF-DNA binding dynamics, we harness the chemical master equation [29] instead of Eq. (1).

In this formulation, $P(n, t)$ denotes the probability that $n$ copies of the TF are occupying the target DNA site at time $t$. If this DNA site can afford at most $N$ copies of the TF at once, $n=$ $0,1, \cdots, N$ and $\sum_{n=0}^{N} P(n, t)=1$. If we further define $P(n, t) \equiv 0$ for $n \neq 0,1, \cdots, N$ and assume that the DNA-binding TFs are hardly accessible by molecular machineries such as for protein degradation, the temporal change of $P(n, t)$ with $n=0,1, \cdots, N$ is governed by this master equation:

$$
\begin{array}{r}
\frac{\partial P(n, t)}{\partial t}=k_{\mathrm{a}} V\left[A_{\mathrm{TF}}(t)-\frac{n-1}{V}\right]\left(B_{\mathrm{DNA}}-\frac{n-1}{V}\right) P(n-1, t)- \\
\left\{k_{\mathrm{a}} V\left[A_{\mathrm{TF}}(t)-\frac{n}{V}\right]\left(B_{\mathrm{DNA}}-\frac{n}{V}\right)+n k_{\delta}\right\} P(n, t)+(n+1) k_{\delta} P(n+1, t),
\end{array}
$$

where $k_{\mathrm{a}}$ and $k_{\delta}$ denote the TF-DNA binding and unbinding rates, respectively [analogous to the parameters in Eq. (1)], $V$ is the nuclear volume, $A_{\mathrm{TF}}(t)$ is the total TF concentration in the nucleus, and $B_{\mathrm{DNA}}$ is the "concentration" of the target DNA site, i.e., $B_{\mathrm{DNA}}=N V^{-1}$. Here, we assume $A_{\mathrm{TF}}(t) \gg B_{\mathrm{DNA}}$ for little stochasticity in $A_{\mathrm{TF}}(t)$ (i.e., $A_{\mathrm{TF}}(t)$ to be uniquely determined at each time $t$ ) and the cell growth is negligible for a steady nuclear volume (i.e., $V$ to remain constant over time).

Introducing a quantity $C_{\mathrm{TF}}(t) \equiv\left\langle n V^{-1}\right\rangle=V^{-1} \sum_{n=0}^{N} n P(n, t)$ to Eq. (26) results in

$$
\begin{array}{r}
\frac{\mathrm{d} C_{\mathrm{TF}}(t)}{\mathrm{d} t}=k_{\mathrm{a}} \sum_{n=0}^{N}\left[A_{\mathrm{TF}}(t)-\frac{n}{V}\right]\left(B_{\mathrm{DNA}}-\frac{n}{V}\right) P(n, t)-k_{\delta} C_{\mathrm{TF}}(t) \\
=k_{\mathrm{a}}\left\{\left[A_{\mathrm{TF}}(t)-C_{\mathrm{TF}}(t)\right]\left[B_{\mathrm{DNA}}-C_{\mathrm{TF}}(t)\right]+\left\langle\left(\frac{n}{V}\right)^{2}\right\rangle-\left\langle\frac{n}{V}\right\rangle^{2}\right\}-k_{\delta} C_{\mathrm{TF}}(t),
\end{array}
$$

where $\left\langle\left(n V^{-1}\right)^{2}\right\rangle=V^{-2} \sum_{n=0}^{N} n^{2} P(n, t)$. Eq. (27) is reminiscent of Eq. (1), when the stochastic fluctuation in the TF binding $\left[\left\langle\left(n V^{-1}\right)^{2}\right\rangle-\left\langle n V^{-1}\right\rangle^{2}\right]$ is negligible. The stochastic fluctuation, 
however, cannot be ignored for small $N$. For simplicity, we will henceforth consider the case of $N=1$ and thus of $B_{\mathrm{DNA}}=V^{-1}$. In this case, Eq. (27) is rewritten as

$$
\frac{\mathrm{d} \bar{C}_{\mathrm{TF}}(\tau)}{\mathrm{d} \tau}=\frac{\bar{A}_{\mathrm{TF}}(\tau)}{K V}-\left[1+\bar{A}_{\mathrm{TF}}(\tau)\right] \bar{C}_{\mathrm{TF}}(\tau)=\left[1+\bar{A}_{\mathrm{TF}}(\tau)\right]\left[\bar{C}_{\mathrm{TFQ}}(\tau)-\bar{C}_{\mathrm{TF}}(\tau)\right]
$$

where $\tau \equiv k_{\delta} t$ and $K \equiv k_{\delta} / k_{\mathrm{a}}$ as adopted in Eq. (2), $\bar{A}_{\mathrm{TF}}(\tau) \equiv A_{\mathrm{TF}}(t) / K, \bar{C}_{\mathrm{TF}}(\tau) \equiv C_{\mathrm{TF}}(t) /$ $K$, and $\bar{C}_{\mathrm{TFQ}}(\tau)$ is given by

$$
\bar{C}_{\mathrm{TFQ}}(\tau) \equiv \frac{\bar{A}_{\mathrm{TF}}(\tau)}{K V\left[1+\bar{A}_{\mathrm{TF}}(\tau)\right]}
$$

In a similar fashion to the tQSSA in Sec. II.A, one may consider an estimate $\bar{C}_{\mathrm{TF}}(\tau) \approx \bar{C}_{\mathrm{TFQ}}(\tau)$, or equivalently, $C_{\mathrm{TF}}(t) \approx C_{\mathrm{TFQ}}(t)$ with this form:

$$
C_{\mathrm{TFQ}}(t) \equiv \frac{A_{\mathrm{TF}}(t)}{V\left[K+A_{\mathrm{TF}}(t)\right]}
$$

$C_{\mathrm{TFQ}}(t)$ looks very similar to the $\mathrm{MM}$ rate law, regarding $B_{\mathrm{DNA}}=V^{-1}$. Nevertheless, $C_{\mathrm{TFQ}}(t)$ is not a mere continuum of Eq. (9), because the denominator in $C_{\mathrm{TFQ}}(t)$ includes $K+A_{\mathrm{TF}}(t)$, but not $K+A_{\mathrm{TF}}(t)+V^{-1}$ from $B_{\mathrm{DNA}}=V^{-1}$. In fact, the discrepancy between $C_{\mathrm{TFQ}}(t)$ and Eq. (9) comes from the inherent stochasticity in the TF-DNA binding dynamics in Eqs. (26) and (27). In this regard, directly relevant to $C_{\mathrm{TFQ}}(t)$ is the stochastic version of the $\mathrm{MM}$ rate law with denominator $K+A(t)+B(t)-V^{-1}$ proposed by Levine and Hwa [30]. $C_{\mathrm{TFQ}}(t)$ is a fundamentally more correct approximant for the DNA-binding TF level with $N=1$ than both $C_{\mathrm{tQ}}(t)$ [Eq. (7)] and its descendant [Eq. (9)].

Still, the use of $C_{\mathrm{TFQ}}(t)$ to estimate $C_{\mathrm{TF}}(t)$ in Eq. (28) stands on the assumption that $C_{\mathrm{TF}}(t)$ approaches the equilibrium fast enough each time before the marked temporal change of $A_{\mathrm{TF}}(t)$. Following a similar procedure to Sec. II.A, we relieve this quasi-steady state assumption and improve the approximation of $C_{\mathrm{TF}}(t)$ in the case of time-varying $A_{\mathrm{TF}}(t)$. Notice that the exact solution of Eq. (28) is

$$
\begin{aligned}
\bar{C}_{\mathrm{TF}}(\tau)=\int_{\tau_{0}}^{\tau}[1 & \left.+\bar{A}_{\mathrm{TF}}\left(\tau^{\prime}\right)\right] \bar{C}_{\mathrm{TFQ}}\left(\tau^{\prime}\right) e^{-\int_{\tau^{\prime}}^{\tau}\left[1+\bar{A}_{\mathrm{TF}}\left(\tau^{\prime \prime}\right)\right] \mathrm{d} \tau^{\prime \prime}} \mathrm{d} \tau^{\prime} \\
& +\bar{C}_{\mathrm{TF}}\left(\tau_{0}\right) e^{-\int_{\tau_{0}}^{\tau}\left[1+\bar{A}_{\mathrm{TF}}\left(\tau^{\prime}\right)\right] \mathrm{d} \tau^{\prime}}
\end{aligned}
$$

where $\tau_{0}$ denotes an arbitrarily assigned, initial point of $\tau$. Assume that $1+\bar{A}_{\mathrm{TF}}\left(\tau^{\prime}\right)$ changes rather slowly over $\tau^{\prime}$ to satisfy

$$
1+\bar{A}_{\mathrm{TF}}\left(\tau^{\prime}\right) \approx 1+\bar{A}_{\mathrm{TF}}(\tau) \text { for } \tau^{\prime} \text { in the range } \tau-\frac{1}{1+\bar{A}_{\mathrm{TF}}(\tau)} \lesssim \tau^{\prime} \leq \tau
$$

As we will show later, Eq. (32) is readily satisfied in physiologically-relevant conditions. With Eq. (32), Eq. (31) for $\tau \gg \tau_{0}+\left[1+\bar{A}_{\mathrm{TF}}\left(\tau_{0}\right)\right]^{-1}$ is approximated as

$$
\bar{C}_{\mathrm{TF}}(\tau) \approx\left[1+\bar{A}_{\mathrm{TF}}(\tau)\right] \int_{-\infty}^{\tau} \bar{C}_{\mathrm{TFQ}}\left(\tau^{\prime}\right) e^{-\left[1+\bar{A}_{\mathrm{TF}}(\tau)\right]\left(\tau-\tau^{\prime}\right)} \mathrm{d} \tau^{\prime} .
$$

The Taylor expansion $\bar{C}_{\mathrm{TFQ}}\left(\tau^{\prime}\right)=\bar{C}_{\mathrm{TFQ}}(\tau)-\left(\tau-\tau^{\prime}\right) \bar{C}_{\mathrm{TFQ}}^{\prime}(\tau)+\left(\tau-\tau^{\prime}\right)^{2} \bar{C}_{\mathrm{TFQ}}^{\prime \prime}(\tau) / 2-\cdots$ leads Eq. (33) to

$$
\begin{gathered}
\bar{C}_{\mathrm{TF}}(\tau) \approx \bar{C}_{\mathrm{TFQ}}(\tau)-\frac{1}{1+\bar{A}_{\mathrm{TF}}(\tau)} \frac{\mathrm{d} \bar{C}_{\mathrm{TFQ}}(\tau)}{\mathrm{d} \tau} \int_{0}^{\infty} x e^{-x} \mathrm{~d} x \\
\quad+\frac{1}{2\left[1+\bar{A}_{\mathrm{TF}}(\tau)\right]^{2}} \frac{\mathrm{d}^{2} \bar{C}_{\mathrm{TFQ}}(\tau)}{\mathrm{d} \tau^{2}} \int_{0}^{\infty} x^{2} e^{-x} \mathrm{~d} x-\cdots \\
=\bar{C}_{\mathrm{TFQ}}(\tau)-\frac{1}{1+\bar{A}_{\mathrm{TF}}(\tau)} \frac{\mathrm{d} \bar{C}_{\mathrm{TFQ}}(\tau)}{\mathrm{d} \tau}+\frac{1}{\left[1+\bar{A}_{\mathrm{TF}}(\tau)\right]^{2}} \frac{\mathrm{d}^{2} \bar{C}_{\mathrm{TFQ}}(\tau)}{\mathrm{d} \tau^{2}}-\cdots \\
=\bar{C}_{\mathrm{TFQe}}(\tau)+\frac{1}{\left[1+\bar{A}_{\mathrm{TF}}(\tau)\right]^{2}} \frac{\mathrm{d}^{2} \bar{C}_{\mathrm{TFQ}}(\tau)}{\mathrm{d} \tau^{2}}-\cdots,
\end{gathered}
$$

where $\bar{C}_{\mathrm{TFQe}}(\tau)$ is defined as

$$
\bar{C}_{\mathrm{TFQ}}(\tau) \equiv \bar{C}_{\mathrm{TFQ}}(\tau)-\frac{1}{1+\bar{A}_{\mathrm{TF}}(\tau)} \frac{\mathrm{d} \bar{C}_{\mathrm{TFQ}}(\tau)}{\mathrm{d} \tau} .
$$

In a similar way to Sec. II.A, we take the Taylor expansion of the time-delayed form of $\bar{C}_{\mathrm{TFQ}}(\tau)$ :

$$
\bar{C}_{\mathrm{TFQ}}\left[\tau-\frac{1}{1+\bar{A}_{\mathrm{TF}}(\tau)}\right]=\bar{C}_{\mathrm{TFQ}}(\tau)-\frac{1}{1+\bar{A}_{\mathrm{TF}}(\tau)} \frac{\mathrm{d} \bar{C}_{\mathrm{TFQ}}(\tau)}{\mathrm{d} \tau}+\frac{1}{2\left[1+\bar{A}_{\mathrm{TF}}(\tau)\right]^{2}} \frac{\mathrm{d}^{2} \bar{C}_{\mathrm{TFQ}}(\tau)}{\mathrm{d} \tau^{2}}-\cdots
$$


Interestingly, the zeroth-order and first-order derivative terms of $\bar{C}_{\mathrm{TFQ}}(\tau)$ on the right-hand side above are identical to $\bar{C}_{\mathrm{TFQ}}(\tau)$, and the second-order derivative term still covers a half of that term in Eq. (34). Hence, we propose the following approximant for $\bar{C}_{\mathrm{TF}}(\tau)$ :

$$
\bar{C}_{\mathrm{TF} \gamma}(\tau) \equiv \bar{C}_{\mathrm{TFQ}}\left[\tau-\frac{1}{1+\bar{A}_{\mathrm{TF}}(\tau)}\right] \text {. }
$$

The corresponding approximant for $C_{\mathrm{TF}}(t)$ is

$$
C_{\mathrm{TF} \gamma}(t) \equiv C_{\mathrm{TFQ}}\left[t-\frac{k_{\delta}^{-1} K}{K+A_{\mathrm{TF}}(t)}\right] .
$$

As will be shown later, the accuracy of the approximant $\bar{C}_{\mathrm{TF} \gamma}(\tau)$ or $C_{\mathrm{TF} \gamma}(t)$ surpasses that of $\bar{C}_{\mathrm{TFQ}}(\tau)$ or $C_{\mathrm{TFQ}}(t)$. On the other hand, Eqs. (37) and (38) are ill-defined for $\tau-$ $\left[1+\bar{A}_{\mathrm{TF}}(\tau)\right]^{-1}<\tau_{0}$ where $\tau_{0}$ is an initial point of $\tau$. In fact, from the last term in Eq. (31), $\tau$ should satisfy $\tau \gg \tau_{0}+\left[1+\bar{A}_{\mathrm{TF}}\left(\tau_{0}\right)\right]^{-1}$ for the application of any rate law [e.g., Eq. (37), (38), or (30)] whose form does not depend on the initial conditions.

To clarify the preconditions for the use of $\bar{C}_{\mathrm{TF} \gamma}(\tau)$ or $C_{\mathrm{TF} \gamma}(t)$, we first revisit the condition in Eq. (32). Applying $1+\bar{A}_{\mathrm{TF}}\left(\tau^{\prime}\right) \approx 1+\bar{A}_{\mathrm{TF}}(\tau)+\left(\tau^{\prime}-\tau\right) \bar{A}_{\mathrm{TF}}^{\prime}(\tau)$ to Eq. (32) gives rise to this condition:

$$
\varepsilon_{\mathrm{TF}}(\tau) \equiv \frac{\bar{A}_{\mathrm{TF}}(\tau)}{\left[1+\bar{A}_{\mathrm{TF}}(\tau)\right]^{2}}\left|\mu_{\mathrm{TF}}(\tau)\right| \ll 1,
$$

where $\mu_{\mathrm{TF}}(\tau) \equiv \bar{A}_{\mathrm{TF}}^{\prime}(\tau) / \bar{A}_{\mathrm{TF}}(\tau)$. Next, the comparison between Eqs. (34) and (36) shows the difference of $\sim \bar{C}_{\mathrm{TFQ}}^{\prime \prime}(\tau) /\left\{2\left[1+\bar{A}_{\mathrm{TF}}(\tau)\right]^{2}\right\}$ and thereby offers this condition:

$$
\varepsilon_{\mathrm{TF} \gamma}(\tau) \equiv \frac{1}{2\left[1+\bar{A}_{\mathrm{TF}}(\tau)\right]^{2} \bar{C}_{\mathrm{TFQ}}\left\{\tau-\left[1+\bar{A}_{\mathrm{TF}}(\tau)\right]^{-1}\right\}}\left|\frac{\mathrm{d}^{2} \bar{C}_{\mathrm{TFQ}}(\tau)}{\mathrm{d} \tau^{2}}\right| \ll 1,
$$

where $\bar{C}_{\mathrm{TFQ}}^{\prime \prime}(\tau)$ is obtained from Eq. (29) as

$$
\frac{\mathrm{d}^{2} \bar{C}_{\mathrm{TFQ}}(\tau)}{\mathrm{d} \tau^{2}}=\frac{\bar{C}_{\mathrm{TFQ}}(\tau)}{1+\bar{A}_{\mathrm{TF}}(\tau)}\left[\frac{1-\bar{A}_{\mathrm{TF}}(\tau)}{1+\bar{A}_{\mathrm{TF}}(\tau)} \mu_{\mathrm{TF}}^{2}(\tau)+\frac{\mathrm{d} \mu_{\mathrm{TF}}(\tau)}{\mathrm{d} \tau}\right] .
$$

In summary, our approximant $\bar{C}_{\mathrm{TF} \gamma}(\tau)$ shall work when Eqs. (39) and (40) are satisfied. As we will show later, Eq. (39) is in fact easy to satisfy and thus only Eq. (40) tends to serve as a key factor for the validity of $\bar{C}_{\mathrm{TF} \gamma}(\tau)$.

On the other hand, the MM-like formula $\bar{C}_{\mathrm{TFQ}}(\tau)$ would be valid in the following condition from Eq. (34), instead of the condition in Eq. (40):

$$
\varepsilon_{\mathrm{TFQ}}(\tau) \equiv \frac{1}{\bar{C}_{\mathrm{TFQ}}(\tau)}\left|\frac{1}{1+\bar{A}_{\mathrm{TF}}(\tau)} \frac{\mathrm{d} \bar{C}_{\mathrm{TFQ}}(\tau)}{\mathrm{d} \tau}-\frac{1}{\left[1+\bar{A}_{\mathrm{TF}}(\tau)\right]^{2}} \frac{\mathrm{d}^{2} \bar{C}_{\mathrm{TFQ}}(\tau)}{\mathrm{d} \tau^{2}}\right| \ll 1,
$$

where $\bar{C}_{\mathrm{TFQ}}^{\prime}(\tau)=\mu_{\mathrm{TF}}(\tau) \bar{C}_{\mathrm{TFQ}}(\tau) /\left[1+\bar{A}_{\mathrm{TF}}(\tau)\right]$ from Eq. (29) and $\bar{C}_{\mathrm{TFQ}}^{\prime \prime}(\tau)$ is given by Eq. (41).

\section{BIOPHYSICAL APPLICATIONS}

In Sec. II, we derived the revised versions of the MM rate law for time-varying concentrations of molecular components. Here, we will apply this revised rate law to a number of biomolecular processes, in comparison with the existing approaches-the tQSSA and SQSSA. In Secs. III.A-III.C, we will focus on the approximation of quantitative dynamical profiles, and in Secs. III.D and III.E on the prediction for qualitatively new dynamical patterns not expected by the existing approaches.

\section{A. Biochemical reaction and transport}

Imagine that substrate $\mathrm{A}$ binds to enzyme $\mathrm{B}$, which catalyzes a metabolic reaction to convert A to another molecule. The formation of the enzyme-substrate complex follows Eq. (1) or its equivalent Eq. (2). In Eq. (1), we set $k_{\delta}=k_{\mathrm{d}}+r_{\mathrm{c}}$ where $r_{\mathrm{c}}$ is interpreted as the catalytic rate constant of the reaction (conventionally written as $k_{\text {cat }}$ in literature), and $k_{\text {loc }}=k_{\text {dlt }}=0$. In this system, the total substrate concentration $A(t)$ changes over time as

which is equivalent to

$$
\frac{\mathrm{d} A(t)}{\mathrm{d} t}=-r_{\mathrm{c}} C(t)
$$




$$
\mu_{\mathrm{A}}(\tau)=-\frac{r_{\mathrm{c}}}{k_{\delta}} \frac{\bar{C}(\tau)}{\bar{A}(\tau)}
$$

$\mu_{\mathrm{A}}(\tau)$ is defined in Sec. II.A as $\mu_{\mathrm{A}}(\tau) \equiv \bar{A}^{\prime}(\tau) / \bar{A}(\tau)$. We further assume that the total enzyme concentration is constant over time $\left[\bar{B}(\tau)=\bar{B}\right.$ and $\mu_{\mathrm{B}}(\tau)=0$ when $\mu_{\mathrm{B}}(\tau) \equiv \bar{B}^{\prime}(\tau) / \bar{B}(\tau)$ as defined in Sec. II.A]. Eqs. (2) and (44) fully determine the time course of the system with given initial conditions and parameters.

Unlike other molecular events that we will consider later, the majority of known metabolic reactions are likely to be modeled by the SQSSA or tQSSA to a sufficient degree, without the need for our proposed formula $C_{\gamma}(t)$ in Eq. (19) [or equivalently, $\bar{C}_{\gamma}(\tau)$ in Eq. (18)]. Indeed, (i) most enzymatic reactions in Table S1 satisfy Eq. (25) as well as Eqs. (20) and (22) (i.e., $\varepsilon_{1}, \varepsilon_{2}, \varepsilon_{\mathrm{tQ}} \ll 1$ in Table S1), although a malate dehydrogenase does not follow Eq. (25) very well when the substrate is oxaloacetate, and (ii) the enzyme levels in Table S1 are generally much lower than the substrate levels, despite two exceptions of malate dehydrogenase and succinate dehydrogenase (fumarate as a substrate). According to the discussion in Sec. II.A, these (i) and (ii) indicate that the tQSSA [relevant to (i)] or SQSSA [relevant to (i) and (ii)] would often suffice for the kinetic modeling of metabolic reactions. Of note, the SQSSA has long been considered as suitable for the modeling of metabolic reactions [1-3]. Yet, as we will analyze below, the malate dehydrogenase kinetics is precisely described by our formula $C_{\gamma}(t)$, whereas the TQSSA and SQSSA perform rather erroneously.

In addition, we examine the case of nutrient transport into a cell, where an "enzyme" is a transporter protein on the cell surface and a substrate is a small molecule nutrient in the extracellular environment. $r_{\mathrm{c}}$ is then interpreted as the uptake rate of a transporter-binding nutrient. We assume that as the cells reproduce, the transporters increase over time with constant $\mu_{\mathrm{B}}(\tau)=\mu_{\mathrm{B}}$, which is equal to the cell growth rate divided by $k_{\delta}$. With this $\mu_{\mathrm{B}}(\tau)$, Eqs. (2) and (44) govern the full kinetics of the nutrient transport. Our analysis of the welldocumented, phosphotransferase system (PTS) in bacterium Escherichia coli reveals that the sQSSA alone would suffice to describe this system without the need for $C_{\gamma}(t)$, as in Table S2 where $\varepsilon_{1}, \varepsilon_{2}, \varepsilon_{\mathrm{tQ}} \ll 1$ and the transporter level is far below the nutrient level.

As mentioned above, Table S1 indicates that oxaloacetate conversion by malate dehydrogenase would be well described by our revised $\mathrm{MM}$ rate law $\left[C_{\gamma}(t)\right]$, compared to the tQSSA and sQSSA. Indeed, the time trajectory of $C_{\gamma}(t)$ shows remarkable agreement with the simulated enzyme-binding substrate levels $[C(t)]$ from Eqs. (2) and (44), after some transient period of $C(t)$ that depends on the initial condition (Fig. 1 and Text S2); meanwhile, the tQSSA is $\sim 0.3-\mathrm{ms}$ more advanced than $C(t)$ in the overall profile and the SQSSA severely overestimates $C(t)$ [Fig. 1(b)]. For example, at $t=1.9 \mathrm{~ms}, C(t) \approx C_{\gamma}(t)=0.13 \mu \mathrm{M}$ and the sQSSA leads to $0.31 \mu \mathrm{M}$, while the tQSSA results in $0.13 \mu \mathrm{M}$ at $t=1.6 \mathrm{~ms}$ [Fig. $1(\mathrm{~b})$ ]. The overall time shift of the tQSSA from $C(t)$ is caused by the discarding of the time delay $\left[\sim k_{\delta}^{-1} \Delta_{\mathrm{tQ}}^{-1 / 2}(t)\right]$ in enzyme-substrate complex formation in the tQSSA, and the overestimation of $C(t)$ by the SQSSA comes from the unlimited enzyme-substrate binding with the increasing enzymes $\{A(t) B(t) /[K+A(t)]\}$ in the sQSSA.

To conclude, our revised MM rate law provides quantitatively better approximation of the above malate dehydrogenase kinetics than the existing approaches, although the latter are likely to be sufficient for the modeling of many metabolic reaction and transport systems. In the case of protein-protein and TF-DNA interactions, the utility of the revised rate law is more prominent, as we will address below. 
(a)

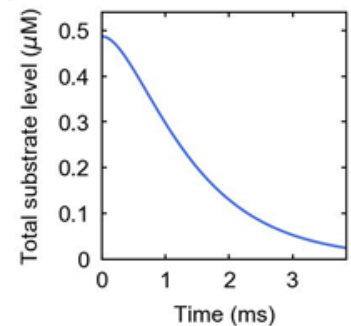

(b)

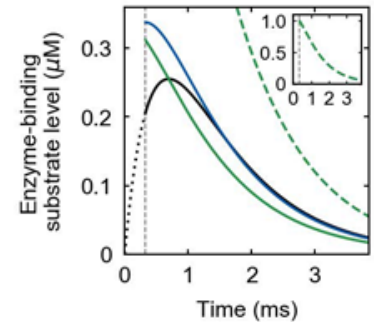

FIG. 1. Oxaloacetate (substrate) conversion by malate dehydrogenase (enzyme). (a) The total substrate concentration over time, calculated by Eqs. (2) and (44) (Text S2). The initial substrate concentration is set as the substrate concentration in Table S1. (b) The enzyme-binding substrate concentrations calculated by Eq. (2) (black solid line; black dotted line for a transient period described below), by our revised MM rate law (blue solid line), by the tQSSA (green solid line), and by the SQSSA (green dashed line) (Text S2). These calculations are based on the total substrate concentration in (a). An inset shows a more complete range of the enzyme-binding substrate concentration from the sQSSA. In (a) and (b), we used the parameters and total enzyme concentration in Table S1. When solving Eqs. (2) and (44), the initial concentration of the enzyme-binding substrate was set to zero. As discussed in Sec. II.A, any form of a rate law without the initial-condition dependency would only work for $t \gg k_{\delta}^{-1} \Delta_{\mathrm{tQ}}^{-1 / 2}(0)$, and also our revised $\mathrm{MM}$ rate law is ill-defined for a period $t<k_{\delta}^{-1} \Delta_{\mathrm{tQ}}^{-1 / 2}(t)$; therefore, for the right comparison with our revised rate law, (b) presents the tQSSA and SQSSA results only after $t=$ $k_{\delta}^{-1} \Delta_{\mathrm{tQ}}^{-1 / 2}(t)$ (vertical dashed line).

\section{B. Protein-protein interaction}

Next, we move to the case of protein-protein interactions. The interacting proteins often show the concentrations comparable with each other [15-17], and thus the tQSSA, not the SQSSA, has recently been recommended for the modeling of their interactions $[12,18]$. Here, we will focus on the interactions between proteins whose abundances oscillate over time with circadian rhythmicity, i.e., $\sim 24$-h periodicity. Circadian protein oscillations play a pivotal role in coordinating numerous physiological processes $[25,26]$. Such time-varying nature in protein abundances might even challenge the relevance of the tQSSA and would serve as a testbed for our revised $\mathrm{MM}$ rate law $\bar{C}_{\gamma}(\tau)$ in Eq. (18).

Suppose that proteins $\mathrm{A}$ and $\mathrm{B}$ have oscillating concentrations with sinusoidal forms:

$$
\bar{A}(\tau)=\bar{A}_{\max }\left\{1-\frac{\alpha_{\mathrm{A}}}{2}\left[1+\cos \left(\frac{2 \pi}{k_{\delta} T} \tau\right)\right]\right\} \text { and } \bar{B}(\tau)=\bar{B}_{\max }\left\{1-\frac{\alpha_{\mathrm{B}}}{2}\left[1+\cos \left(\frac{2 \pi}{k_{\delta} T} \tau-\varphi_{\mathrm{B}}\right)\right]\right\} \text {, }
$$

where $\bar{A}_{\max }\left(\bar{B}_{\max }\right), \alpha_{\mathrm{A}(\mathrm{B})}, T$, and $\varphi_{\mathrm{B}}$ denote the peak level of $\bar{A}(\tau)[\bar{B}(\tau)]$, the peak-totrough difference of $\bar{A}(\tau)[\bar{B}(\tau)]$ divided by the peak level, the oscillation period of a circadian or diurnal rhythm, and the phase difference between $\bar{A}(\tau)$ and $\bar{B}(\tau)$, respectively. Here, $\alpha_{\mathrm{A}}$ and $\alpha_{\mathrm{B}}$ range from 0 to 1 (the closer they are to 1 , the stronger the oscillations) and $0 \leq \varphi_{\mathrm{B}} \leq$ $\pi$ without loss of generality. Throughout this study, we choose $T=24 \mathrm{~h}$. Based on $\bar{A}(\tau)$ and $\bar{B}(\tau)$ in Eq. (45), we numerically solve Eq. (2) to obtain $\bar{C}(\tau)$ (Text S2), and evaluate how well $\bar{C}(\tau)$ is approximated by each of $\bar{C}_{\gamma}(\tau)$, the tQSSA $\bar{C}_{\mathrm{tQ}}(\tau)$ in Eq. (5), and the SQSSA in Eq. (9).

As illustrated in Fig. 2(a), we observe that $\bar{C}_{\gamma}(\tau)$ tends to better match the temporal trajectory of $\bar{C}(\tau)$ than the tQSSA and SQSSA. For systematic evaluation, we define $\phi_{\gamma}^{t}, \phi_{\mathrm{tQ}}^{t}$, and $\phi_{S Q}^{t}$ as the phase differences in hours between $\bar{C}_{\gamma}(\tau)$ and $\bar{C}(\tau)$, between the tQSSA and $\bar{C}(\tau)$, and between the SQSSA and $\bar{C}(\tau)$, respectively (Text S2). The sign of a given phase difference is assigned positive (negative) if the corresponding trajectory has a more advanced (delayed) phase than $\bar{C}(\tau)$. We observe that the signs of $\phi_{\mathrm{tQ}}^{t}$ and $\phi_{\mathrm{SQ}}^{t}$ are always positive and the sign of $\phi_{\gamma}^{t}$ is mostly negative. In the example of Fig. $2(\mathrm{a}), \phi_{\gamma}^{t}=-1.0 \mathrm{~h}, \phi_{\mathrm{tQ}}^{t}=3.05 \mathrm{~h}$, 
$\phi_{\mathrm{sQ}}^{t}=3.1 \mathrm{~h}$, and hence $\left|\phi_{\gamma}^{t}\right|$ is smaller than $\left|\phi_{\mathrm{tQ}}^{t}\right|$ and $\left|\phi_{\mathrm{SQ}}^{t}\right|$. We did find that $\left|\phi_{\gamma}^{t}\right|$ tends to be smaller than $\left|\phi_{\mathrm{tQ}}^{t}\right|$ and $\left|\phi_{\mathrm{sQ}}^{t}\right|$ across physiologically-relevant conditions [Fig. 2(b) and $P<$ $10^{-4}$; see Table S3 and Text S2]. Remarkably, when $\left|\phi_{\gamma}^{t}\right|,\left|\phi_{\mathrm{tQ}}^{t}\right|$, or $\left|\phi_{\mathrm{SQ}}^{t}\right|$ is $\geq 1 \mathrm{~h}$, most parameter conditions (86.2\%) have $\left|\phi_{\gamma}^{t}\right|$ less than both $\left|\phi_{\mathrm{tQ}}^{t}\right|$ and $\left|\phi_{\mathrm{sQ}}^{t}\right|$ at least by one hour, and some of them (22.9\%) even at least by two hours [Figs. 2(c) and 2(d); $P<10^{-4}$ and Text S2]. These findings establish the tendency that $\bar{C}_{\gamma}(\tau)$ is more likely to resemble $\bar{C}(\tau)$ in the phase than the tQSSA and SQSSA.

(a)

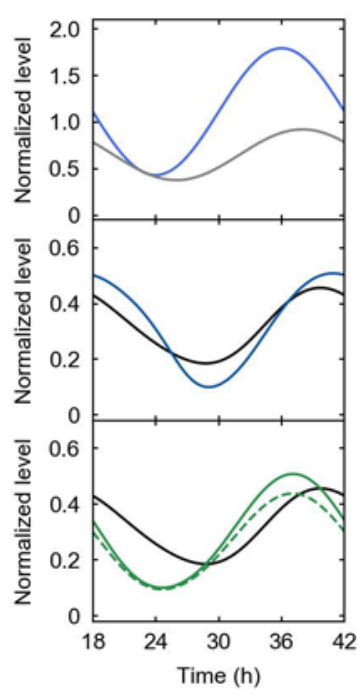

(d)

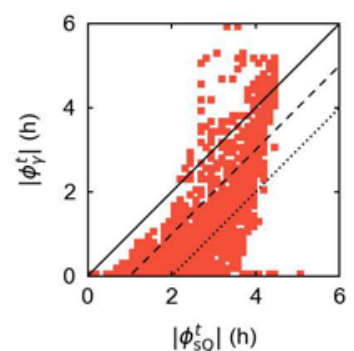

(e)

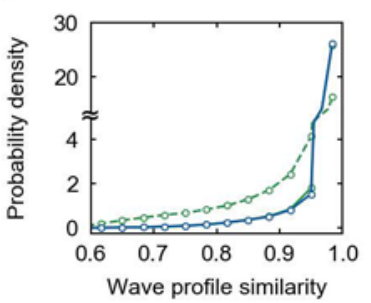

(b)

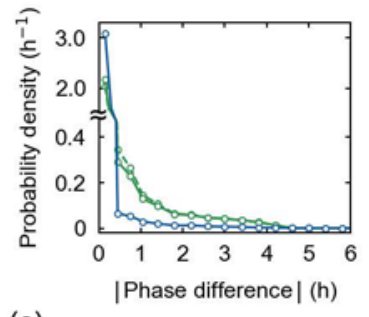

(c)

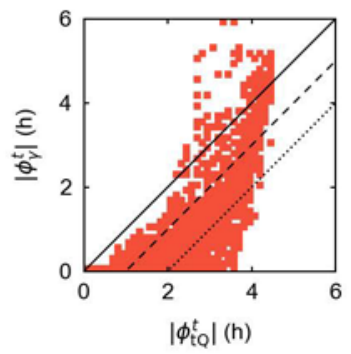

(f)

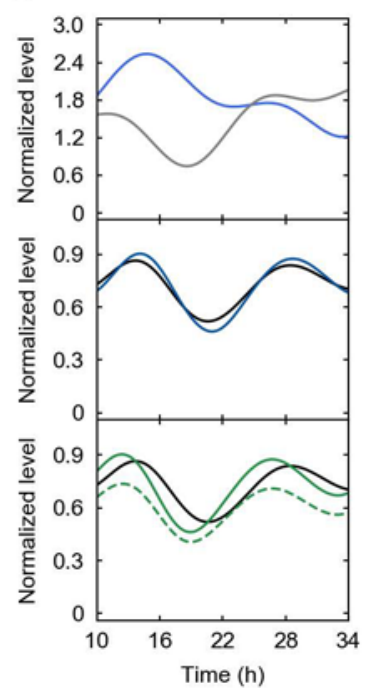

FIG. 2. Protein-protein interaction modeling. (a) Example time series of $\bar{A}(\tau)$ (bluish) and $\bar{B}(\tau)$ (gray) at the top, $\bar{C}(\tau)$ (black) and $\bar{C}_{\gamma}(\tau)$ (blue) at the center, and $\bar{C}(\tau)$ (black), the tQSSA $\bar{C}_{\mathrm{tQ}}(\tau)$ (green solid line), and the sQSSA (green dashed line) at the bottom. The calculations are based on Eqs. (2), (5), (9), (18), and (45), and $t=k_{\delta}^{-1} \tau$ as defined in Sec. II.A. (b) Probability distributions of $\left|\phi_{\gamma}^{t}\right|$ (blue solid line), $\left|\phi_{\mathrm{tQ}}^{t}\right|$ (green solid line), and $\left|\phi_{\mathrm{sQ}}^{t}\right|$ (green dashed line) over randomly-sampled parameter sets in Table S3. (c,d) Scatter plot of $\left|\phi_{\mathrm{tQ}}^{t}\right|$ and $\left|\phi_{\gamma}^{t}\right|$ (c), or that of $\left|\phi_{\mathrm{sQ}}^{t}\right|$ and $\left|\phi_{\gamma}^{t}\right|$ (d), when $\left|\phi_{\gamma}^{t}\right|,\left|\phi_{\mathrm{tQ}}^{t}\right|$, or $\left|\phi_{\mathrm{sQ}}^{t}\right|$ is $\geq 1$ $\mathrm{h}$ with randomly-sampled parameter sets in Table S3. A solid diagonal line corresponds to $\left|\phi_{\gamma}^{t}\right|=\left|\phi_{\mathrm{tQ}}^{t}\right|$ (c) or $\left|\phi_{\gamma}^{t}\right|=\left|\phi_{\mathrm{sQ}}^{t}\right|$ (d), a dashed diagonal line to $\left|\phi_{\gamma}^{t}\right|=\left|\phi_{\mathrm{tQ}}^{t}\right|-1 \mathrm{~h}(\mathrm{c})$ or $\left|\phi_{\gamma}^{t}\right|=\left|\phi_{\mathrm{sQ}}^{t}\right|-1 \mathrm{~h}(\mathrm{~d})$, and a dotted diagonal line to $\left|\phi_{\gamma}^{t}\right|=\left|\phi_{\mathrm{tQ}}^{t}\right|-2 \mathrm{~h}$ (c) or $\left|\phi_{\gamma}^{t}\right|=\left|\phi_{\mathrm{sQ}}^{t}\right|-2 \mathrm{~h}$ (d). Although not covered in (b) and (d), $\left|\phi_{\mathrm{sQ}}^{t}\right|>6 \mathrm{~h}$ for a tiny portion of the parameter sets $(0.03 \%)$, in which still $\left|\phi_{\gamma}^{t}\right|,\left|\phi_{\mathrm{tQ}}^{t}\right| \leq 1 \mathrm{~h}$. (e) 
Probability distributions of $S_{\gamma}$ (blue solid line), $S_{\mathrm{tQ}}$ (green solid line), and $S_{\mathrm{sQ}}$ (green dashed line) over randomly-sampled parameter sets in Table S3. (f) When based on Eq. (46), example time series of $\bar{A}(\tau)$ (bluish) and $\bar{B}(\tau)$ (gray) at the top, $\bar{C}(\tau)$ (black) and $\bar{C}_{\gamma}(\tau)$ (blue) at the center, and $\bar{C}(\tau)$ (black), the tQSSA $\bar{C}_{\mathrm{tQ}}(\tau)$ (green solid line), and the sQSSA (green dashed line) at the bottom. They were calculated in the same way as (a), except for the use of Eq. (46) instead of Eq. (45). For the detailed methods in (a)-(f) and the parameters in (a) and (f), refer to Text S2.

Other than phases, wave profiles (determined by the waveforms and peak levels) are the important features of oscillatory molecular behaviors. Therefore, we define similarity $S_{\gamma}$ between the profiles of $\bar{C}_{\gamma}(\tau)$ and $\bar{C}(\tau)$ by aligning their phases to the same (Text S2). $S_{\gamma}$ is devised to approach 1 away from 0 , as the two wave profiles quantitatively better match each other. We also define the similarity measures $S_{\mathrm{tQ}}$ and $S_{\mathrm{SQ}}$ for the tQSSA and $\bar{C}(\tau)$ and for the sQSSA and $\bar{C}(\tau)$, respectively (Text S2). In the example of Fig. 2(a), $S_{\gamma}=0.86, S_{\mathrm{tQ}}=0.86$, and $S_{\mathrm{SQ}}=0.83$. Based on Eq. (18), one can expect that the main difference between $\bar{C}_{\gamma}(\tau)$ and $\bar{C}_{\mathrm{tQ}}(\tau)$ would be attributed to their phases, rather than to their shapes. As expected, $S_{\gamma}$ and $S_{\mathrm{tQ}}$ tend to be almost equal to each other [Spearman's $\rho=0.89$ and $P<10^{-4}$; see Fig. 2(e) and Text S2]. On the other hand, consistent with the previous suggestions that the TQSSA is more accurate than the sQSSA [12], we found that $S_{\mathrm{sQ}}$ tends to be below $S_{\gamma}$ and $S_{\mathrm{tQ}}$ [Fig. 2(e); $P<10^{-4}$ and Text S2]. Together, $\bar{C}_{\gamma}(\tau)$ and the tQSSA better approximate the wave profile of $\bar{C}(\tau)$ than the SQSSA.

To summarize, our revised rate law $\bar{C}_{\gamma}(\tau)$ tends to serve as a good approximant for $\bar{C}(\tau)$, with a more accurate phase than the tQSSA and SQSSA's and with a wave profile as accurate as the tQSSA's. One may expect that such a high accuracy of $\bar{C}_{\gamma}(\tau)$ compared to the tQSSA's might be indicated by the range of its valid conditions in Eqs. (20), (22), and (23). In fact, most of the physiologically-relevant conditions in Table S3 (88.1\%) satisfy both $\max _{\tau}\left[\varepsilon_{1}(\tau)\right] \leq 0.1$ and $\max _{\tau}\left[\varepsilon_{2}(\tau)\right] \leq 0.1$, and therefore only $\varepsilon_{\gamma}(\tau)$ and $\varepsilon_{\mathrm{tQ}}(\tau)$ remain the key determinants of the validities of $\bar{C}_{\gamma}(\tau)$ and the tQSSA, respectively. Our analysis reveals that $\max _{\tau}\left[\varepsilon_{\gamma}(\tau)\right] \leq$ 0.1 for $55.9 \%$ of the simulated conditions and $\max _{\tau}\left[\varepsilon_{\mathrm{tQ}}(\tau)\right] \leq 0.1$ for $45.4 \%$ of the same conditions, supporting the wider applicability of $\bar{C}_{\gamma}(\tau)$ than the tQSSA's. Naturally, $\max _{\tau}\left[\varepsilon_{\gamma}(\tau)\right]$ and $\max _{\tau}\left[\varepsilon_{\mathrm{tQ}}(\tau)\right]$ correlate positively with $\left|\phi_{\gamma}^{t}\right|$ and $\left|\phi_{\mathrm{tQ}}^{t}\right|$, respectively (Spearman's $\rho=0.52$ between $\max _{\tau}\left[\varepsilon_{\gamma}(\tau)\right]$ and $\left|\phi_{\gamma}^{t}\right|$ with $P<10^{-4}$, and $\rho=0.74$ between $\max _{\tau}\left[\varepsilon_{\mathrm{tQ}}(\tau)\right]$ and $\left|\phi_{\mathrm{tQ}}^{t}\right|$ with $P<10^{-4}$; see Text S2).

Although physiologically less relevant, the oscillatory protein levels with irregular rhythmicity may provide another testbed for the approximating capability of $\bar{C}_{\gamma}(\tau)$. Hence, instead of Eq. (45), we consider the following $\bar{A}(\tau)$ and $\bar{B}(\tau)$ and numerically solve Eq. (2):

$$
\begin{gathered}
\bar{A}(\tau)=\frac{1}{N} \sum_{i=1}^{N} \bar{A}_{\max , i}\left\{1-\frac{\alpha_{\mathrm{A}, i}}{2}\left[1+\cos \left(\frac{2 \pi}{k_{\delta} T_{\mathrm{A}, i}} \tau-\varphi_{\mathrm{A}, i}\right)\right]\right\}, \\
\bar{B}(\tau)=\frac{1}{N} \sum_{i=1}^{N} \bar{B}_{\max , i}\left\{1-\frac{\alpha_{\mathrm{B}, i}}{2}\left[1+\cos \left(\frac{2 \pi}{k_{\delta} T_{\mathrm{B}, i}} \tau-\varphi_{\mathrm{B}, i}\right)\right]\right\} .
\end{gathered}
$$

We further choose $N=10$ and randomly select the other parameters from the ranges in Text S2. Still, we find that $\bar{C}_{\gamma}(\tau)$ tends to better approximate even such an irregular profile of $\bar{C}(\tau)$ than the tQSSA and SQSSA, as illustrated in Fig. 2(f).

Next, we move to a real-world example of oscillating-protein interactions. In plant Arabidopsis thaliana, ZEITLUPE (ZTL) is an essential protein for a normal circadian periodicity. ZTL is stabilized by a direct interaction with another protein GIGANTEA (GI), and this interaction is enhanced by blue light [31-33]. As a result, ZTL protein levels oscillate in lightdark cycles, despite the constitutive mRNA expression of ZTL [31]. We here assess how well our revised MM rate law accounts for the experimental ZTL profile over time, through the modeling of the ZTL-GI interaction. If $A(t)$ and $B(t)$ represent the ZTL and GI concentrations, respectively, then the ZTL turnover dynamics can be described by the following equation: 


$$
\frac{\mathrm{d} A(t)}{\mathrm{d} t}=g_{\mathrm{A}}-r_{\mathrm{c}} C(t)-r_{\mathrm{A}}[A(t)-C(t)],
$$

where $g_{\mathrm{A}}$ is the ZTL synthesis rate, $C(t)$ is the concentration of ZTL-GI complex, and $r_{\mathrm{A}}$ and $r_{\mathrm{C}}$ denote the degradation rates of free ZTL and GI-binding ZTL, respectively. $r_{\mathrm{c}}<r_{\mathrm{A}}$ because $\mathrm{GI}$ stabilizes ZTL. We assume $k_{\delta}=k_{\mathrm{d}}+r_{\mathrm{c}}$ and $C(t)$ is determined by Eq. (1). Because blue light enhances the ZTL-GI interaction, we assume that $k_{\mathrm{d}}$ and $K$ in light do not exceed $k_{\mathrm{d}}$ and $K$ in darkness, respectively. We set $B(t)$ as the known GI profile [Fig. 3(a)] [31] multiplied by a scaling coefficient $w_{\mathrm{GI}}$, because the original $\mathrm{GI}$ profile is given by the concentration levels at a relative scale, not at the absolute scale. For the same reason, when comparing $A(t)$ with the experimental ZTL profile [Fig. 3(a)] [31], we use $A_{\mathrm{S}}(t) \equiv A(t) / w_{\mathrm{ZTL}}$ where $w_{\mathrm{ZTL}}$ is another scaling coefficient.

(a)

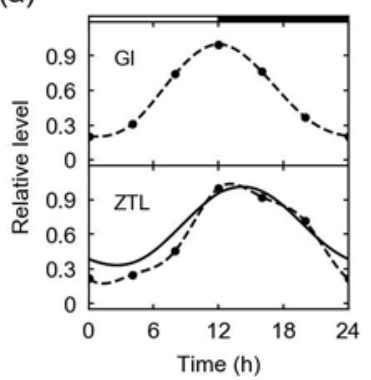

(c)

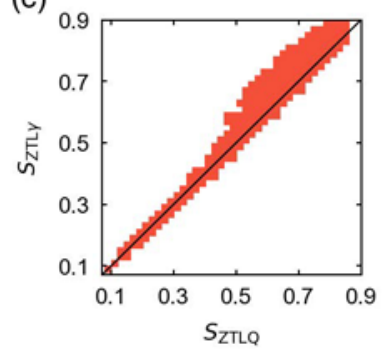

(b)

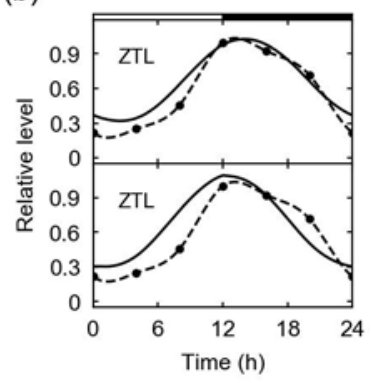

(d)

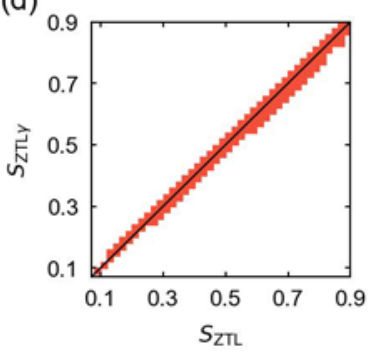

FIG. 3. ZTL-GI interaction in Arabidopsis. (a) The experimental GI levels (dots) [31] and their interpolation (dashed line) at the top, and the experimental ZTL levels (dots) [31], their interpolation (dashed line), and version (i)-based $A_{\mathrm{s}}(t)$ (solid line) at the bottom. (b) The experimental ZTL levels (dots) and their interpolation (dashed line) in (a), together with $A_{s}(t)$ (solid line) from version (ii) at the top, or version (iii) at the bottom. In (a) and (b), $A_{\mathrm{s}}(t)$ and versions (i)-(iii) are defined in Sec. III.B and were computed with a given parameter set in Text S2. Horizontal white and black segments in (a) and (b) correspond to light and dark intervals, respectively. (c,d) Scatter plot of $S_{\mathrm{ZTLQ}}$ and $S_{\mathrm{ZTL} \gamma}$ (c), or that of $S_{\mathrm{ZTL}}$ and $S_{\mathrm{ZTL} \gamma}$ (d), over randomly-selected parameter sets in Table S4. A diagonal line corresponds to $S_{\mathrm{ZTL} \gamma}=S_{\mathrm{ZTLQ}}$ (c) or $S_{\mathrm{ZTL} \gamma}=S_{\mathrm{ZTL}}$ (d). For the detailed methods in (a)-(d), refer to Text S2.

We compute three different versions of $A(t)$ for their comparison with the experimental ZTL profile: (i) the first version is the solution of $A(t)$ from Eqs. (1) and (47), (ii) the second version is the solution of only Eq. (47) with the replacement of $C(t)$ by $C_{\gamma}(t)$ in Eq. (19), and (iii) the last version is similar to version (ii), but with the replacement of $C(t)$ by $C_{\mathrm{tQ}}(t)$ in Eq. (7). In other words, version (i) is the full modeling result that we treat as the gold standard to assess the relative accuracies of versions (ii) and (iii) from our revised MM rate law and the tQSSA, respectively. We do not longer consider the SQSSA because the tQSSA has already proven to be more accurate than the SQSSA in the previous studies $[12,13,18-24]$ as well as in our analysis above (Sec. II.A. and Figs. 1 and 2).

We compute versions (i)-(iii) for randomly-selected parameters $g_{\mathrm{A}}, r_{\mathrm{A}}, r_{\mathrm{c}}, w_{\mathrm{GI}}$, and $w_{\mathrm{ZTL}}$ with $k_{\mathrm{d}}$ and $K$ in light and darkness (Table $\mathrm{S} 4$ and Text $\mathrm{S} 2$ ). We define similarity $S_{\mathrm{ZTL}}$ between $A_{\mathrm{S}}(t)$ and the empirical ZTL profile when $A_{\mathrm{S}}(t)$ is calculated from version (i) by the above 
relation $A_{\mathrm{S}}(t)=A(t) / w_{\mathrm{ZTL}}$ (Text S2). $S_{\mathrm{ZTL}}$ is devised to approach 1 away from 0 , as $A_{\mathrm{S}}(t)$ quantitatively better matches the ZTL profile. Analogously, $S_{\mathrm{ZTL} \gamma}$ and $S_{\mathrm{ZTLQ}}$ are defined for the cases with versions (ii) and (iii), respectively (Text S2). Figs. 3(a) and $3(\mathrm{~b})$ present an example that $A_{\mathrm{S}}(t)$ from version (ii) is as close to the experimental ZTL profile as $A_{\mathrm{S}}(t)$ from version $(i)$, and is closer to that experimental profile than $A_{\mathrm{S}}(t)$ from version (iii) $\left(S_{\mathrm{ZTL}}=0.88\right.$, $S_{\mathrm{ZTL} \gamma}=0.88$, and $\left.S_{\mathrm{ZTLQ}}=0.79\right)$. Indeed, most of our simulated conditions (78.2\%) show $S_{\mathrm{ZTL} \gamma}$ higher than $S_{\mathrm{ZTLQ}}$ [Fig. 3(c); $P<10^{-4}$ and Text S2], while $S_{\mathrm{ZTL} \gamma}$ and $S_{\mathrm{ZTL}}$ are almost the same as each other through the simulated conditions [Fig. 3(d)]. We hence conclude that our revised $\mathrm{MM}$ rate law is comparable with the full kinetic modeling in quantitative accounting for the experimental ZTL profile, with an improvement on the tQSSA.

\section{TF-DNA interaction}

We now examine the case of TF-DNA interactions. Suppose that the TF concentration oscillates over time in a sinusoidal form:

$$
\bar{A}_{\mathrm{TF}}(\tau)=\bar{A}_{\max }\left\{1-\frac{\alpha_{\mathrm{A}}}{2}\left[1+\cos \left(\frac{2 \pi}{k_{\delta} T} \tau\right)\right]\right\},
$$

where $\bar{A}_{\text {max }}, \alpha_{\mathrm{A}}$, and $T$ are defined the same as in Eq. (45). Based on $\bar{A}_{\mathrm{TF}}(\tau)$ in Eq. (48), we numerically solve Eq. (28) to obtain $\bar{C}_{\mathrm{TF}}(\tau)$ (Text S2), and evaluate how well $\bar{C}_{\mathrm{TF}}(\tau)$ is approximated by our formula $\bar{C}_{\mathrm{TF} \gamma}(\tau)$ in Eq. (37) or by the MM-like formula $\bar{C}_{\mathrm{TFQ}}(\tau)$ in Eq. (29). Regarding $\bar{C}_{\mathrm{TFQ}}(\tau)$, note that the distinction between the tQSSA and SQSSA is meaningless here because of condition $A_{\mathrm{TF}}(t) \gg B_{\mathrm{DNA}}$ in Sec. II.B.

(a)

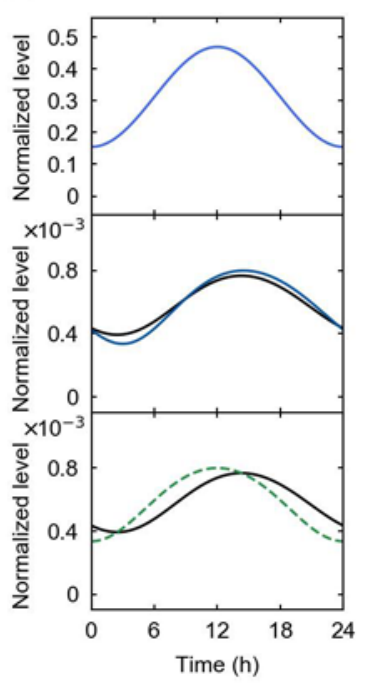

(d)

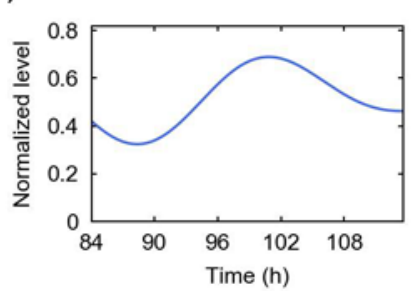

(b)

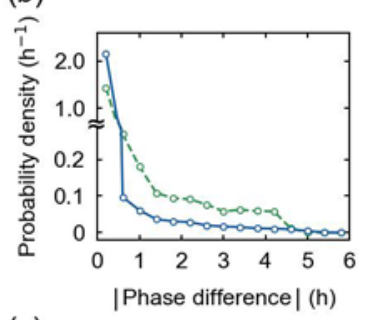

(c)
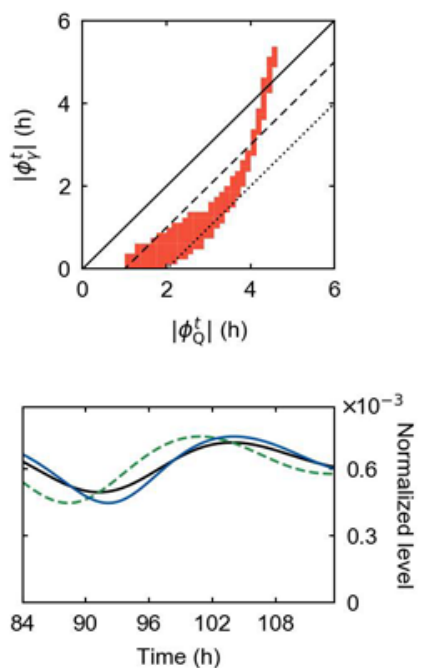

FIG. 4. TF-DNA interaction modeling. (a) Example time series of $\bar{A}_{\mathrm{TF}}(\tau)$ at the top, $\bar{C}_{\mathrm{TF}}(\tau)$ (black) and $\bar{C}_{\mathrm{TF} \gamma}(\tau)$ (blue) at the center, and $\bar{C}_{\mathrm{TF}}(\tau)$ (solid line) and $\bar{C}_{\mathrm{TFQ}}(\tau)$ (dashed line) at the bottom. The calculations are based on Eqs. (28), (29), (37), and (48), and $t=k_{\delta}^{-1} \tau$ as defined in Sec. II.B. (b) Probability distributions of $\left|\phi_{\gamma}^{t}\right|$ (solid line) and $\left|\phi_{Q}^{t}\right|$ (dashed line) over randomly-sampled parameter 
sets in Table S3. (c) Scatter plot of $\left|\phi_{Q}^{t}\right|$ and $\left|\phi_{\gamma}^{t}\right|$ when $\left|\phi_{Q}^{t}\right|$ or $\left|\phi_{\gamma}^{t}\right| \geq 1 \mathrm{~h}$ with randomly-sampled parameter sets in Table S3. A solid diagonal line corresponds to $\left|\phi_{\gamma}^{t}\right|=\left|\phi_{\mathrm{Q}}^{t}\right|$, a dashed diagonal line to $\left|\phi_{\gamma}^{t}\right|=\left|\phi_{\mathrm{Q}}^{t}\right|-1 \mathrm{~h}$, and a dotted diagonal line to $\left|\phi_{\gamma}^{t}\right|=\left|\phi_{\mathrm{Q}}^{t}\right|-2 \mathrm{~h}$. (d) When based on Eq. (49), example time series of $\bar{A}_{\mathrm{TF}}(\tau)$ on the left and $\bar{C}_{\mathrm{TF}}(\tau)$ (black solid line), $\bar{C}_{\mathrm{TF} \gamma}(\tau)$ (blue solid line), and $\bar{C}_{\mathrm{TFQ}}(\tau)$ (green dashed line) on the right. They were calculated in the same way as (a), except for the use of Eq. (49) instead of Eq. (48). For the detailed methods in (a)-(d) and the parameters in (a) and (d), refer to Text S2.

As illustrated in Fig. 4(a), we observe that $\bar{C}_{\mathrm{TF} \gamma}(\tau)$ tends to better match the time trajectory of $\bar{C}_{\mathrm{TF}}(\tau)$ than $\bar{C}_{\mathrm{TFQ}}(\tau)$. In a similar fashion to Sec. III.B, we use quantities $\phi_{\gamma}^{t}$ and $\phi_{\mathrm{Q}}^{t}$ as the phase differences in hours between $\bar{C}_{\mathrm{TF} \gamma}(\tau)$ and $\bar{C}_{\mathrm{TF}}(\tau)$, and between $\bar{C}_{\mathrm{TFQ}}(\tau)$ and $\bar{C}_{\mathrm{TF}}(\tau)$, respectively (Text S2). The magnitudes of these phase differences reach up to $\sim 5 \mathrm{~h}$ in physiologically-relevant parameter conditions [Fig. 4(b) and Table S3]. The sign of a given phase difference is assigned positive (negative) if the corresponding trajectory has a more advanced (delayed) phase than $\bar{C}_{\mathrm{TF}}(\tau)$. In the simulated conditions (Table S3), we observe $\phi_{\mathrm{Q}}^{t} \geq 0$ and $\phi_{\gamma}^{t} \leq 0$. In the example of Fig. 4(a), $\phi_{\gamma}^{t}=-0.3 \mathrm{~h}$ and $\phi_{\mathrm{Q}}^{t}=2.3 \mathrm{~h}$, and here $\left|\phi_{\gamma}^{t}\right|$ is smaller than $\left|\phi_{\mathrm{Q}}^{t}\right|$. Indeed, our analysis suggests that $\left|\phi_{\gamma}^{t}\right|$ tends to be smaller than $\left|\phi_{\mathrm{Q}}^{t}\right|$ over the physiologically-relevant conditions [Fig. 4(b); $P<10^{-4}$ and Text S2]. When $\left|\phi_{\gamma}^{t}\right|$ or $\left|\phi_{\mathrm{Q}}^{t}\right|$ is $\geq 1 \mathrm{~h}$, most parameter conditions (91.6\%) have $\left|\phi_{\gamma}^{t}\right|$ less than $\left|\phi_{\mathrm{Q}}^{t}\right|$ at least by one hour, and a quarter of them even at least by two hours [Fig. 4(c); $P<10^{-4}$ and Text S2]. These results suggest that $\bar{C}_{\mathrm{TF} \gamma}(\tau)$ is more likely to resemble $\bar{C}_{\mathrm{TF}}(\tau)$ in its phase than $\bar{C}_{\mathrm{TFQ}}(\tau)$ is.

Unlike the cases of phases, we expect that the wave profiles predicted by $\bar{C}_{\mathrm{TF} \gamma}(\tau)$ and $\bar{C}_{\mathrm{TFQ}}(\tau)$ would be almost the same, with regards to Eq. (37). We examine this issue in a similar way to Sec. III.B: we measure similarity $S_{\gamma}$ between the profiles of $\bar{C}_{\mathrm{TF} \gamma}(\tau)$ and $\bar{C}_{\mathrm{TF}}(\tau)$ after aligning their phases to the same (Text S2). Analogously, $S_{\mathrm{Q}}$ is defined for $\bar{C}_{\mathrm{TFQ}}(\tau)$ and $\bar{C}_{\mathrm{TF}}(\tau)$. $S_{\gamma}$ and $S_{\mathrm{Q}}$ range from 0 to 1: the larger a value of $S_{\gamma}\left(S_{\mathrm{Q}}\right)$, the more similar are the wave profiles of $\bar{C}_{\mathrm{TF} \gamma}(\tau)\left[\bar{C}_{\mathrm{TFQ}}(\tau)\right]$ and $\bar{C}_{\mathrm{TF}}(\tau)$ (Text S2). As anticipated, $S_{\gamma}$ and $S_{\mathrm{Q}}$ take almost the same values as each other (Spearman's $\rho=0.94$ and $P<10^{-4}$ ) and both are $>0.7$ for the physiologically-relevant conditions (Text S2).

To summarize, our revised MM rate law $\bar{C}_{\mathrm{TF} \gamma}(\tau)$ improves a phase approximation of $\bar{C}_{\mathrm{TF}}(\tau)$, while $\bar{C}_{\mathrm{TF} \gamma}(\tau)$ and $\bar{C}_{\mathrm{TFQ}}(\tau)$ exhibit very similar wave profiles. One may expect that such a higher accuracy of $\bar{C}_{\mathrm{TF} \gamma}(\tau)$ in the phases might be indicated by the range of the valid conditions of $\bar{C}_{\mathrm{TF} \gamma}(\tau)$ in Eqs. (39) and (40). In fact, most of our simulated conditions (92.0\%) in Table S3 satisfy $\max _{\tau}\left[\varepsilon_{\mathrm{TF}}(\tau)\right] \leq 0.1$ and therefore only $\varepsilon_{\mathrm{TF} \gamma}(\tau)$ and $\varepsilon_{\mathrm{TFQ}}(\tau)$ in Eqs. (40) and (42) remain the key determinants of the validities of $\bar{C}_{\mathrm{TF} \gamma}(\tau)$ and $\bar{C}_{\mathrm{TFQ}}(\tau)$, respectively. Our analysis shows that $\max _{\tau}\left[\varepsilon_{\mathrm{TF} \gamma}(\tau)\right] \leq 0.1$ for $81.6 \%$ of the conditions, slightly more than the conditions (69.9\%) with $\max _{\tau}\left[\varepsilon_{\mathrm{TFQ}}(\tau)\right] \leq 0.1$. Naturally, $\max _{\tau}\left[\varepsilon_{\mathrm{TF} \gamma}(\tau)\right]$ and $\max _{\tau}\left[\varepsilon_{\mathrm{TFQ}}(\tau)\right]$ strongly correlate with $\left|\phi_{\gamma}^{t}\right|$ and $\left|\phi_{\mathrm{Q}}^{t}\right|$, respectively (Spearman's $\rho=0.69$ between $\max _{\tau}\left[\varepsilon_{\mathrm{TF} \gamma}(\tau)\right]$ and $\left|\phi_{\gamma}^{t}\right|$, and $\rho=0.89$ between $\max _{\tau}\left[\varepsilon_{\mathrm{TFQ}}(\tau)\right]$ and $\left|\phi_{\mathrm{Q}}^{t}\right| ; P<$ $10^{-4}$ and Text S2).

Although physiologically less relevant, the oscillatory TF level with irregular rhythmicity may provide another testbed for the approximating capability of $\bar{C}_{\mathrm{TF} \gamma}(\tau)$. Hence, instead of Eq. (48), we consider the following $\bar{A}_{\mathrm{TF}}(\tau)$ and numerically solve Eq. (28):

$$
\bar{A}_{\mathrm{TF}}(\tau)=\frac{1}{N} \sum_{i=1}^{N} \bar{A}_{\mathrm{max}, i}\left\{1-\frac{\alpha_{\mathrm{A}, i}}{2}\left[1+\cos \left(\frac{2 \pi}{k_{\delta} T_{\mathrm{A}, i}} \tau-\varphi_{\mathrm{A}, i}\right)\right]\right\},
$$

We choose $N=10$ and randomly select the other parameters from the ranges in Text S2. Even with such irregularity of the rhythms, $\bar{C}_{\mathrm{TF} \gamma}(\tau)$ is still found to improve the approximation of $\bar{C}_{\mathrm{TF}}(\tau)$ compared to $\bar{C}_{\mathrm{TFQ}}(\tau)$, as illustrated in Fig. $4(\mathrm{~d})$. 


\section{Mammalian circadian clock}

The quantitatively more accurate results from our revised $\mathrm{MM}$ rate law than from the previous approaches motivate us to ask the following question: can the revised MM rate law predict qualitatively new patterns of a dynamical system beyond its quantitative characterization? We will answer this question through the study of the mammalian circadian system.

The core part of the mammalian circadian clock harbors a transcriptional/post-translational negative feedback loop that generates autonomous protein oscillations with circadian rhythmicity [25,34]. Heterodimers of CLOCK and BMAL1 proteins activate the transcription of Period (Per) and Cryptochrome (Cry) genes, and the encoded PER and CRY proteins form PERCRY complexes that are translocated to the nucleus. In the nucleus, they interact with CLOCKBMAL1 complexes to inhibit the CLOCK-BMAL1 transcriptional activities. These positive (CLOCK and BMAL1) and negative (PER and CRY) arms constitute the negative feedback loop for circadian oscillations in protein levels and activities.

A previous study suggests that the tQSSA for the interaction between activator (CLOCKBMAL1) and repressor (PER-CRY) leads to more natural rhythm generation than the SQSSA, because the tQSSA captures the ultrasensitive response of the repressor's transcription to the activator's or repressor's concentration-that is, a large change in the transcription rate from a small change in the activator or repressor level [18]. This ultrasensitive response, which is manifested by small $K$ and balanced activator and repressor levels, amplifies rhythms and prevents their dampening $[18,20]$. Here, we will show that our revised MM rate law further captures the intrinsic time delays in the protein-protein and protein-DNA assembly formation and thereby predicts the rhythmic patterns not expected by the tQSSA.

For the modeling of the mammalian clock, we interpret $A(t), B(t)$, and $C(t)$ in Eq. (1) as the concentrations of activator, repressor, and their complex in the nucleus, respectively. For simplicity, we assume the constancy of $A(t)$, i.e., $A(t)=A$ as the activator's oscillation is weaker than the repressor's and dispensable for the circadian rhythmicity [34-37]. The resulting model comprises Eq. (1) with $A(t)=A$ and the following equations modified from a previous model [18]:

$$
\begin{gathered}
\frac{\mathrm{d} M(t)}{\mathrm{d} t}=a_{0} C_{\mathrm{TF}}(t)-b_{0} M(t), \quad(50) \\
\frac{\mathrm{d} B_{\mathrm{cy}}(t)}{\mathrm{d} t}=a_{1} M(t)-b_{1} B_{\mathrm{cy}}(t), \quad(51) \\
\frac{\mathrm{d} B(t)}{\mathrm{d} t}=a_{2} B_{\mathrm{cy}}(t)-r_{\mathrm{f}}[B(t)-C(t)]-r_{\mathrm{c}} C(t), \quad(52) \\
\frac{\mathrm{d} C_{\mathrm{TF}}(t)}{\mathrm{d} t}=k_{\mathrm{TFa}}[A-C(t)]\left[\frac{1}{V}-C_{\mathrm{TF}}(t)\right]-k_{\mathrm{TF} \delta} C_{\mathrm{TF}}(t) .
\end{gathered}
$$

Here, $M(t), B_{\mathrm{cy}}(t)$, and $C_{\mathrm{TF}}(t)$ are the concentrations of repressor mRNA, cytoplasmic repressor protein, and activator on repressor's promoter, respectively. Eq. (53) is equivalent to Eq. (28) in its content. $a_{0}, a_{1}$, and $a_{2}$ denote the transcription, translation, and translocation rates of the repressor, respectively. $b_{1}$ represents the sum of the translocation and degradation rates of the repressor in the cytoplasm, and thus satisfies $b_{1} N_{\mathrm{c}}>a_{2}$ where $N_{\mathrm{c}}$ is the cytoplasm-to-nucleus volume ratio. $b_{0}, r_{\mathrm{f}}$, and $r_{\mathrm{c}}$ are the degradation rates of repressor mRNA, free repressor protein, and activator-binding repressor protein, respectively. By definition, $r_{\mathrm{c}}$ satisfies $r_{\mathrm{c}}<k_{\delta}$ for $k_{\delta}$ in Eq. (1). $k_{\mathrm{TFa}}, k_{\mathrm{TF} \delta}$, and $V$ are the same as $k_{\mathrm{a}}, k_{\delta}$, and $V$ in Eqs. (26)-(28), respectively. To be precise, $A-C(t)$ in Eqs. (1) and (53) should be replaced by $A-C(t)-C_{\mathrm{TF}}(t)$; however, this replacement does not much affect our simulation results, and we thus keep Eqs. (1) and (53) for the straightforward use of approximants for $C(t)$ and $C_{\mathrm{TF}}(t)$ as will be demonstrated later.

Our model simulation with Eqs. (1) and (50)-(53) leads to the oscillation of the variables in a subset of the parameter conditions in Table S5 (Text S2). For comparison, we test another model with our revised MM rate law. This model consists only of Eqs. (50)-(52) where $C(t)$ and $C_{\mathrm{TF}}(t)$ are replaced by $C_{\gamma}(t)$ and $C_{\mathrm{TF} \gamma}(t)$ in Eqs. (19) and (38), respectively, and $A-$ $C_{\gamma}(t)$ corresponds to $A_{\mathrm{TF}}(t), k_{\mathrm{TF} \delta}$ to $k_{\delta}$, and $k_{\mathrm{TF} \delta} / k_{\mathrm{TFa}}$ to $K$ in Eqs. (30) and (38). Likewise, 
the tQSSA- and SQSSA-based models are constructed by using the approximants in Eqs. (7) and (9) instead of $C_{\gamma}(t)$, respectively, and using $C_{\mathrm{TFQ}}(t)$ in Eq. (30) instead of $C_{\mathrm{TF} \gamma}(t)$.
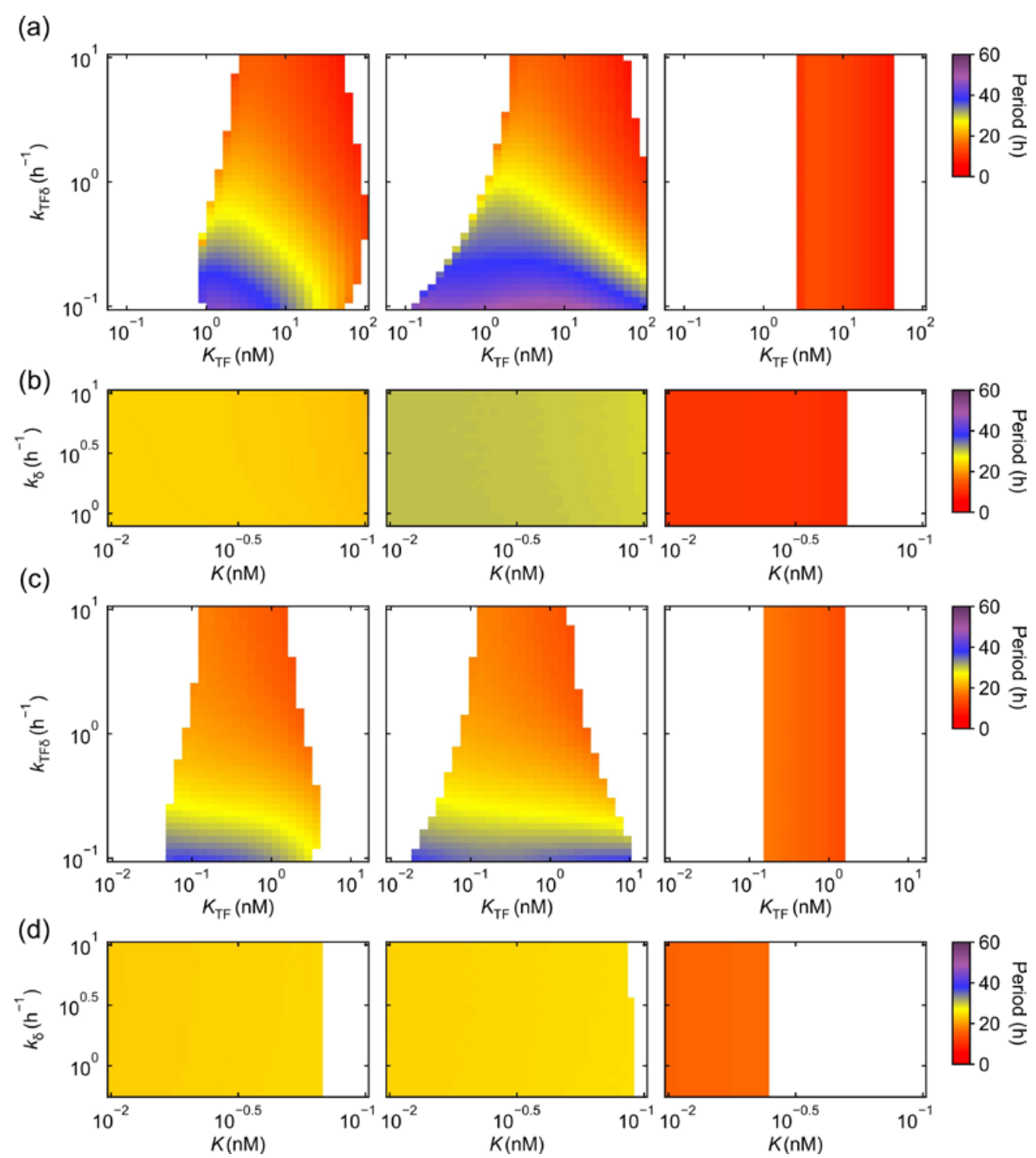

FIG. 5. Mammalian clock simulation with varying parameters. In (a)-(d), the parameter ranges with observed oscillations from each simulation scheme are colored by their oscillation periods, according to the color scale on the rightmost side. (a) and (b) have the same parameters except $K_{\mathrm{TF}}\left(K_{\mathrm{TF}} \equiv\right.$ $\left.k_{\mathrm{TF} \delta} k_{\mathrm{TFa}}^{-1}\right), k_{\mathrm{TF} \delta}, K$, and $k_{\delta}$. Likewise, (c) and (d) have the same parameters except $K_{\mathrm{TF}}, k_{\mathrm{TF} \delta}, K$, and $k_{\delta}$. The simulation results from the full model with Eqs. (1) and (50)-(53) (left), from our revised MM rate law (center), and from the tQSSA (right) are presented over the ranges of $K_{\mathrm{TF}}$ and $k_{\mathrm{TF} \delta}$ in (a) and (c), or the ranges of $K$ and $k_{\delta}$ in (b) and (d), while the other parameters are fixed. For the simulation methods and parameters in (a)-(d), refer to Sec. III.D and Text S2.

Consistent with the previous work and the characteristics of the well-studied Goodwin model $[18,38]$, the SQSSA-based model fails to produce oscillations for any simulated conditions (Table S5 and Text S2). On the other hand, the tQSSA-based model generates oscillations as previously demonstrated [18], but with substantial deviations from the exact model simulation with Eqs. (1) and (50)-(53) (Fig. 5 and Text S2). For example, a decrease in $k_{\mathrm{TF} \delta}$ in the exact model simulation tends to facilitate the development of oscillations to some extent by widening the oscillatory range of $K_{\mathrm{TF}} \equiv k_{\mathrm{TF} \delta} k_{\mathrm{TFa}}^{-1}$, and concurrently lengthens the oscillation period [Figs. 5(a) and 5(c)]; the tQSSA-based model does not reproduce these 
patterns, though [Figs. 5(a) and 5(c)]. Besides, the exact simulation results exhibit the oscillations over the range of $K$ far beyond small $K$ values from the tQSSA-based results [Figs. 5(b) and 5(d)]. Moreover, the tQSSA-based oscillation periods are limited to short periods compared to the exact simulation results (Fig. 5).

In contrast, the simulation with our revised MM rate law shows good agreement with the exact model simulation in terms of the overall patterns of oscillation onset and periods (Fig. 5 and Text S2). For example, the revised MM rate law predicts the periods of 13.6 to $49.6 \mathrm{~h}$ with varying $k_{\mathrm{TF} \delta}$ at $K_{\mathrm{TF}}=11.9 \mathrm{nM}$ in Fig. 5(a), and the exact periods span 13.0 to $33.4 \mathrm{~h}$ in the same conditions. Meanwhile, the tQSSA-based period is limited to $11.6 \mathrm{~h}$ there [Fig. 5(a)]. Regarding the revised rate law, the wide range of the oscillatory parameters and the period variations comparable to the exact simulation results comes from the time-delay terms in protein-protein and protein-DNA assembly formation in Eqs. (19) and (38). These intrinsic time-delay effects, which are absent in the tQSSA, enhance the rhythmicity of the dynamics and lengthen the oscillation periods. A caveat is that the revised rate law tends to rather overestimate the oscillatory parameter ranges compared to the exact model simulation (Fig. 5). Aside from this caveat, the revised rate law accounts for the overall qualitative and quantitative outcomes of the mammalian clock model not expected by the tQSSA or SQSSA.

\section{E. Rhythmic degradation of proteins}

Our revised MM rate law bears the potential to offer analytical insights into biomolecular dynamics otherwise hardly addressable. We demonstrate this potential through the analysis of circadian protein degradation. Some circadian clock proteins are not only rhythmically produced but also decompose with rhythmic degradation rates [Figs. 6(a) and 6(b)] [39-43]. Recently, we have suggested that the rhythmic degradation rates of proteins with circadian production can spontaneously emerge without any explicitly time-dependent regulatory mechanism of the degradation processes $[39,44]$. If the rhythmic degradation rate peaks at the descending phase of the protein profile and stays relatively low elsewhere, it is supposed to save much of the biosynthetic cost in maintaining a circadian rhythm [39,42]. Can our revised $\mathrm{MM}$ rate law explain this inherent rhythmicity in the degradation rates of circadian proteins?

In the following analysis, $A(t), B(t)$, and $C(t)$ represent the concentrations of substrate protein, its rate-limiting proteolytic mediator (e.g., E3 ubiquitin ligase, or kinase in the case of phospho-dependent ubiquitination), and their complex, respectively. The protein turnover dynamics is described by Eq. (1) and the following equation:

$$
\frac{\mathrm{d} A(t)}{\mathrm{d} t}=g(t)-r(t) A(t)
$$

where $g(t)$ and $r(t)$ are the protein synthesis (translation) and degradation rates, respectively. Here, $r(t) A(t)=r_{\mathrm{c}} C(t)$ with substrate turnover rate $r_{\mathrm{c}}$ upon the complex formation and $k_{\delta}=k_{\mathrm{d}}+r_{\mathrm{c}}$ in Eq. (1). Straightforwardly,

$$
r(t)=r_{\mathrm{c}} \frac{C(t)}{A(t)} \text {. }
$$

To exclude the possibility of the time-dependent regulation of the degradation process, we assume the constancy of $B(t)$ in Eq. (1) with $B(t)=B$. Given the circadian profile of protein synthesis rate $g(t)$, the numerical solution of Eqs. (1), (54), and (55) gives rise to the degradation rate $r(t)$ (Text S2). In this calculation, we use the sinusoidal form of $g(t)$ :

$$
g(t)=g_{\max }\left\{1-\frac{\alpha_{\mathrm{g}}}{2}\left[1+\cos \left(\frac{2 \pi t}{T}\right)\right]\right\}
$$

where $g_{\text {max }}, \alpha_{\mathrm{g}}$, and $T$ are constants.

On the other hand, our revised MM rate law $C_{\gamma}(t)$ and the tQSSA $C_{\mathrm{tQ}}(t)$ in Eqs. (7) and (19) provide the following approximants for $r(t)$ :

$$
r_{\gamma}(t) \equiv r_{\mathrm{c}} \frac{C_{\gamma}(t)}{A(t)} \text { and } r_{\mathrm{tQ}}(t) \equiv r_{\mathrm{c}} \frac{C_{\mathrm{tQ}}(t)}{A(t)}
$$


In addition, the SQSSA-based approximant for $r(t)$ is $r_{\mathrm{c}} B /[K+A(t)]$, although not of focus here because the tQSSA is more accurate than the SQSSA as discussed above. In the case of oscillating protein profile $A(t)$, one may expect that rhythmicity in the degradation rate is readily realized by the tQSSA or SQSSA, because the tQSSA- or SQSSA-based degradation rate shows the $A(t)$-dependency such as $r_{\mathrm{c}} B /[K+A(t)]$ in the sQSSA. However, the tQSSA- or sQSSA-based degradation rate is a decreasing function of $A(t)$, which does not match the experimental degradation patterns in Figs. $6(\mathrm{a})$ and $6(\mathrm{~b})$. Moreover, the degradation rhythmicity from the tQSSA or SQSSA is only manifested when the substrate protein becomes saturated, e.g., when $A(t)$ approaches or exceeds $K+B$ in the tQSSA.

(a)

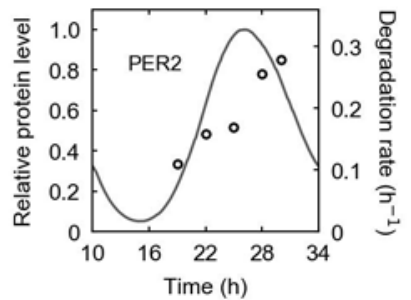

(c)

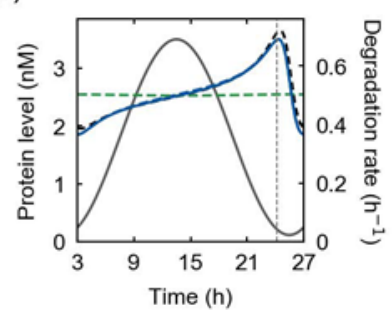

(b)

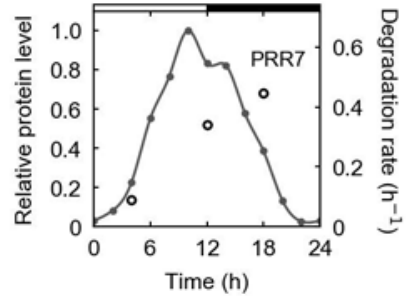

(d)

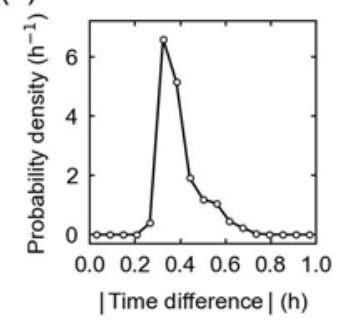

FIG. 6. Rhythmic degradation of circadian proteins. (a) The experimental abundance levels (solid line) and degradation rates (open circles) of the mouse PERIOD2 (PER2) protein [40]. (b) The experimental abundance levels (dots, interpolated by a solid line) and degradation rates (open circles) of PSEUDO RESPONSE REGULATOR 7 (PRR7) protein in Arabidopsis $[41,42,50]$. Horizontal white and black segments correspond to light and dark intervals, respectively. (c) Simulated protein abundance $A(t)$ (gray solid line) and degradation rate $r(t)$ (black dashed line), along with the approximate degradation rates $r_{\gamma}(t)$ (blue solid line), $r_{\gamma 2}(t)$ (blue dashed line), and $r_{\mathrm{tQ}}(t)$ (green dashed line) from Eqs. (1) and (54)-(58). $r_{\gamma}(t)$ and $r_{\gamma_{2}}(t)$ highly overlap and are not visually distinguishable here. A vertical dashed line corresponds to the peak time of $-A^{\prime}(t) / A(t)$. The simulation was performed with a given parameter set in Text S2. (d) The probability distribution of the peak-time difference between $r(t)$ and $-A^{\prime}(t) / A(t)$ over randomly-sampled parameter sets in Table S6. For the detailed methods in (a)-(d), refer to Text $\mathbf{S 2}$.

In contrast, our revised MM rate law naturally accounts for the experimental degradation patterns through the inherent time delay in protein complex formation. For mathematical simplicity, we consider condition $\max _{t}[A(t)] \ll B$, and then $r_{\gamma}(t)$ in Eq. (57) becomes approximated by

$$
r_{\gamma}(t) \sim r_{\gamma 2}(t) \equiv \frac{r_{\mathrm{c}} B}{K+B} \frac{A\left(t-k_{\delta}^{-1} \frac{K}{K+B}\right)}{A(t)}=\frac{r_{\mathrm{c}} B}{K+B}\left\{1-\frac{k_{\delta}^{-1} K}{K+B}\left[\frac{1}{A(t)} \frac{\mathrm{d} A(t)}{\mathrm{d} t}\right]+\cdots\right\} .
$$

In this condition, the tQSSA does not predict rhythmicity in the degradation rate, as $r_{\mathrm{tQ}}(t)$ in Eq. (57) is roughly constant $\left[r_{\mathrm{tQ}}(t) \sim r_{\mathrm{c}} B /(K+B)\right]$. On the other hand, $r_{\gamma 2}(t)$ in Eq. (58) is an approximately increasing function of $-A^{\prime}(t) / A(t)$, and thus our revised MM rate law predicts that the degradation rate would increase as time goes from the ascending to descending phases of the protein profile. This tendency in degradation rhythmicity is consistent with the experimental observation of mammalian and plant clock proteins in Figs. $6(\mathrm{a})$ and $6(\mathrm{~b})$. This rhythmicity can be explained by an unsynchronized interplay between protein translation and 
degradation processes [39] due to their inter-event time delay. For example, in the case of protein ubiquitination, ubiquitin ligases with a finite binding affinity would not always capture all newly-translated substrates, and therefore, a lower proportion of the substrates may be ubiquitinated during the ascending phase of the substrate profile than during the descending phase. The degradation rate hence tends to be lower at times other than the descending phase. One may expect that this effect would be enhanced with more limited ubiquitin ligases, under the condition when the substrate level with circadian production undergoes a steeply rising and falling oscillation. This expectation is supported by Eq. (58), where the amplitude of $r_{\gamma 2}(t)$ is roughly proportional to $K /(K+B)$ as well as to the amplitude of $-A^{\prime}(t) / A(t)$. Eq. (58) further predicts that the degradation rate would peak around the peak time of $-A^{\prime}(t) / A(t)$.

In the example of Fig. 6(c), the degradation rate $r(t)$ computed fully from Eqs. (1) and (54)(56) does exhibit the rhythmic profile in excellent agreement with our predictions $\left[r_{\gamma}(t)\right.$ and $r_{\gamma_{2}}(t)$ in Eqs. (57) and (58)], compared to the tQSSA [ $r_{\mathrm{tQ}}(t)$ in Eq. (57)]. In fact, for the parameter conditions of $\max _{t}[A(t)] \ll B$, the simulated $r_{\mathrm{tQ}}(t)$ is found to be almost constant as previously anticipated, even though we focus on markedly rhythmic $r(t)\left\{\max _{t}[r(t)]-\right.$ $\left.\min _{t}[r(t)]\right\} / \max _{t}[r(t)]>0.4$; Table S6 and Text S2\}. We next define similarity $S_{\mathrm{r} \gamma}$ between $r_{\gamma}(t)$ and $r(t)$, so that $S_{\mathrm{r} \gamma}$ approaches 1 away from 0 as the profiles of $r_{\gamma}(t)$ and $r(t)$ are similar to each other (Text S2). Likewise, we define similarity $S_{\text {r } \gamma 2}$ between $r_{\gamma 2}(t)$ and $r(t)$. All our simulated conditions with rhythmic $r(t)$ and $\max _{t}[A(t)] \ll B$ show $S_{\mathrm{r} \gamma}, S_{\mathrm{r} \gamma 2}>0.97$ $\left(P<10^{-4}\right.$; Table S6 and Text S2), indicating that both $r_{\gamma}(t)$ and $r_{\gamma 2}(t)$ well approximate $r(t)$ in these simulated conditions.

Notably, the peak time of $r(t)$ in Fig. $6(\mathrm{c})$ is $24.5 \mathrm{~h}$, close to $24.2 \mathrm{~h}$ of the maximum $-A^{\prime}(t) / A(t)$ as predicted in Eq. (58). Indeed, the peaks of the $r(t)$ profiles show only $\leq 0.8 \mathrm{~h}$ time differences from the maximum $-A^{\prime}(t) / A(t)$ values across the simulated conditions [Fig. 6(d); Table 56 and Text S2], supporting our prediction from Eq. (58). Together, our revised MM rate law provides a useful analytical framework of the rhythmic degradation of circadian proteins, which is hardly addressable by the conventional ways.

\section{PARAMETER ESTIMATION}

An accurate function of variables and parameters is required for good parameter estimation based on the fitting of the function $[13,45,46]$. In this regard, we compare the accuracies of parameters estimated from our revised MM rate law and the tQSSA by their fitting to the full model simulation results. Specifically, we consider protein-protein interactions with timevarying protein concentrations $A(t)$ and $B(t)$ from Eq. (45) by $A(t)=K \bar{A}(\tau), B(t)=K \bar{B}(\tau)$, $t=k_{\delta}^{-1} \tau$, and parameters set as in Text S2. Given these profiles $A(t)$ and $B(t)$ and the parameters $K$ and $k_{\delta}, C(t)$ is determined by Eq. (1) and we treat this $C(t)$ as the "true" concentration of protein complex. To the profile of $C(t)$, we then fit our revised MM rate law [ $C_{\gamma}(t)$ in Eq. (19)] or the tQSSA [ $C_{\mathrm{tQ}}(t)$ in Eq. (7)] based on the given $A(t)$ and $B(t)$, and estimate the original parameters of $C(t)$ : the fitting of $C_{\gamma}(t)$ estimates both $K$ and $k_{\delta}$, and the fitting of $C_{\mathrm{tQ}}(t)$ estimates only $K$ (Text S2). Because the tQSSA has already been reported for its better parameter-estimation capability than the SQSSA's $[12,46]$, we here do not evaluate the case of the SQSSA. In this work, the parameters were estimated by Powell's method [47], and an alternative method hardly changed our results (Text S2).

Likewise, we consider TF-DNA interactions with time-varying TF concentration $A_{\mathrm{TF}}(t)$ from Eq. (48) by $A_{\mathrm{TF}}(t)=K \bar{A}_{\mathrm{TF}}(\tau), t=k_{\delta}^{-1} \tau$, and parameters set as in Text S2. Given the profile $A_{\mathrm{TF}}(t), B_{\mathrm{DNA}}\left(=V^{-1}\right)$, and the kinetic parameters $K$ and $k_{\delta}, C_{\mathrm{TF}}(t)$ is determined by Eq. (28) and we treat this $C_{\mathrm{TF}}(t)$ as the "true" concentration of TF-DNA assembly. To the profile of $C_{\mathrm{TF}}(t)$, we then fit $C_{\mathrm{TF} \gamma}(t)$ in Eq. (38) and $C_{\mathrm{TFQ}}(t)$ in Eq. (30) based on the given $A_{\mathrm{TF}}(t)$ and $V^{-1}$, and estimate the original parameters of $C_{\mathrm{TF}}(t)$ : the fitting of $C_{\mathrm{TF} \gamma}(t)$ estimates both $K$ 
and $k_{\delta}$, and the fitting of $C_{\mathrm{TFQ}}(t)$ estimates only $K$ (Text S2). Like the above protein-protein interaction case, we used Powell's method to estimate the parameters [47], while an alternative method did not much affect our results (Text S2).

In the case of protein-protein interactions, Fig. 7(a) reveals that the use of our revised rate law improves parameter estimation compared to the tQSSA, as $C_{\gamma}(t)$ tends to give a more accurate estimate of $K$ than $C_{\mathrm{tQ}}(t)$. For example, in the cases that the relative error of $K$ estimated from $C_{\mathrm{tQ}}(t)$ is $\geq 0.2$, most of the $C_{\gamma}(t)$-based estimates (75.9\%) show the relative error $<0.2\left(P<10^{-4}\right.$ and Text S2), and even their $65.9 \%$ show the relative error $<0.1$ [Fig. 7(a)]. In the case of TF-DNA interactions, our revised MM rate law allows a comparably weak improvement in the estimation of $K$, though [Fig. $7(\mathrm{~b})$ ].

(a)

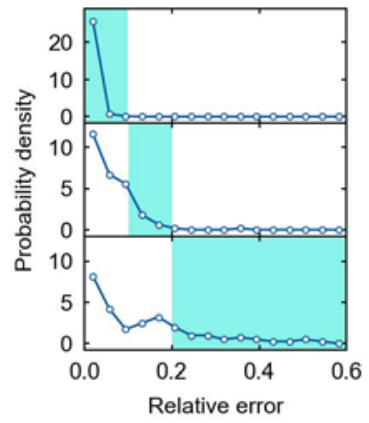

(c)

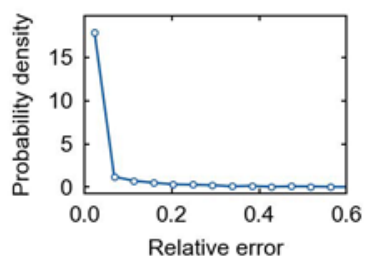

(b)

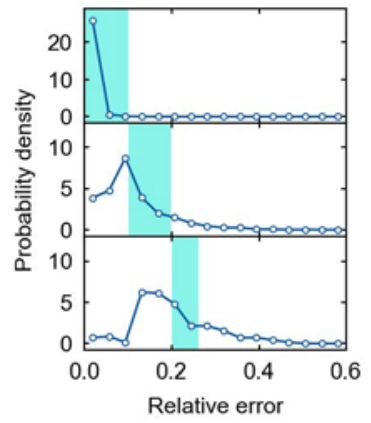

(d)

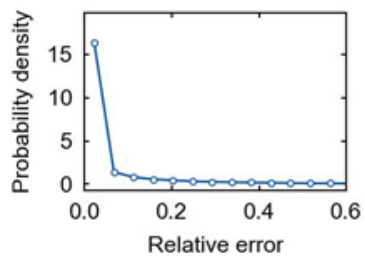

FIG. 7. Parameter estimation for protein-protein and TF-DNA interaction models. (a) The probability distribution of the relative error of estimated $K$ for a protein-protein interaction model in Eqs. (1) and (45). The estimation was performed by the fitting of $C_{\gamma}(t)$ [Eq. (19)] to $C(t)$ [Eq. (1)], when the relative error of the estimated $K$ from $C_{\mathrm{tQ}}(t)$ [Eq. (7)] is $<0.1$ (top), $\geq 0.1$ and $<0.2$ (center), or $\geq 0.2$ (bottom). (b) The probability distribution of the relative error of estimated $K$ for a TF-DNA interaction model in Eqs. (28) and (48). The estimation was performed by the fitting of $C_{\mathrm{TF} \gamma}(t)$ [Eq. (38)] to $C_{\mathrm{TF}}(t)$ [Eq. (28)], when the relative error of the estimated $K$ from $C_{\mathrm{TFQ}}(t)$ [Eq. (30)] is $<0.1$ (top), $\geq 0.1$ and $<0.2$ (center), or $\geq 0.2$ (bottom). In (a) and (b), shaded is the actually-observed range of the relative error of the estimated $K$ from $C_{\mathrm{tQ}}(t)$ (a) or $C_{\mathrm{TFQ}}(t)$ (b) across our simulated conditions (Text S2). More than a half of the simulated conditions show that the relative error of the estimated $K$ from $C_{\gamma}(t)$ (a) or $C_{\mathrm{TF} \gamma}(t)$ (b) is $<0.1$ (top and center) or $<0.2$ (bottom). (c) The probability distribution of the relative error of $k_{\delta}$ estimated by $C_{\gamma}(t)$ for the protein-protein interaction model used in (a). (d) The probability distribution of the relative error of $k_{\delta}$ estimated by $C_{\mathrm{TF} \gamma}(t)$ for the TF-DNA interaction model used in (b). Although not shown in (c) and (d), there exist a negligible portion of the simulated conditions where the relative error of the estimated $k_{\delta}$ is $>0.6$. For the detailed methods in (a)-(d), refer to Text S2.

Unlike $K, k_{\delta}$ can only be estimated through $C_{\gamma}(t)$ and $C_{\mathrm{TF} \gamma}(t)$, and hence the comparison to its estimates from $C_{\mathrm{tQ}}(t)$ and $C_{\mathrm{TFQ}}(t)$ is not possible. Of note, $k_{\delta}$ is found to have the relative error $<0.1$ for most of the $C_{\gamma}(t)$ - and $C_{\mathrm{TF} \gamma}(t)$-based estimates, $86.6 \%$ and $80.7 \%$, respectively [Figs. 7(c) and 7(d)]. 


\section{CONCLUSION AND DISCUSSION}

In this study, we proposed the revised MM rate law, which improves the description of molecular interaction dynamics with time-varying molecular concentrations. When applied to protein-protein and TF-DNA interaction dynamics, our revised MM rate law tends to capture the correct phases of the oscillatory profiles, compared to the existing rate laws. The revised rate law also improves the relevant parameter estimation. In the case of the mammalian circadian clock, the revised rate law well accounts for the overall patterns of oscillation onsets and periods from the full model simulations, which are not captured by the tQSSA or SQSSA. Notwithstanding the apparent complexity of our revised rate law, it provides valuable analytical insights into the spontaneous establishment and temporal profiles of the rhythmic degradation rates of circadian proteins, hardly addressable by the previous rate laws. This demonstrated predictability of our revised MM rate law for the dynamics of various model and empirical systems originates from the rigorously derived, time-delay effects in the molecular complex formation.

Further elaboration and physical interpretation of our theoretical framework, in concert with extensive experimental profiling of molecular complexes in regulatory or signaling pathways $[15,16,31-33]$, are warranted for correct understanding and modeling of the interplay between cellular components and its functional consequences. Although the mammalian and plant data presented here are supportive of our theoretical predictions, experimental tests are clearly warranted including direct validation of the proposed rate law. This validation could involve the measurement of the time series of molecular complex concentrations by coimmunoprecipitation assays or other techniques, and high-temporal resolution data are preferred for the comparison with the proposed rate law. Besides, comprehensive consideration of stochastic fluctuation and nonlinearity in molecular binding events $[29,48,49]$ would be needed for more complete development of our theory, although the stochasticity in TF-DNA interactions was partially reflected in this work.

\section{REFERENCES}

1. V. Henri, Lois Générales de l'Action des Diastases (Librairie Scientifique A. Hermann, 1903).

2. L. Michaelis and M. L. Menten, Die Kinetik der Invertinwirkung, Biochem. Z. 49, 333-369 (1913).

3. G. E. Briggs and J. B. S. Haldane, A Note on the Kinetics of Enzyme Action, Biochem. J. 19, 338 (1925).

4. J. Gunawardena, Time-Scale Separation-Michaelis and Menten's Old Idea, Still Bearing Fruit, FEBS. J. 281, 473-488 (2014).

5. C. Gérard and A. Goldbeter, A Skeleton Model for the Network of Cyclin-Dependent Kinases Driving the Mammalian Cell Cycle, Interface Focus 1, 24-35 (2011).

6. K. C. Chen, A. Csikasz-Nagy, B. Gyorffy, J. Val, B. Novak, and J. J. Tyson, Kinetic Analysis of a Molecular Model of the Budding Yeast Cell Cycle, Mol. Biol. Cell 11, 369-391 (2000).

7. J. C. Leloup and A. Goldbeter, Toward a Detailed Computational Model for the Mammalian Circadian Clock, Proc. Natl. Acad. Sci. USA 100, 7051-7056 (2003).

8. J. Garcia-Ojalvo, Modeling Gene Expression in Time and Space, Annu. Rev. Biophys. 42, 605-627 (2013).

9. M. Foo, D. E. Somers, and P.-J. Kim, Kernel Architecture of the Genetic Circuitry of the Arabidopsis Circadian System, PLoS Comput. Biol. 12, e1004748 (2016).

10. T. D. Pollard, A Guide to Simple and Informative Binding Assays, Mol. Biol. Cell 21, 4061-4067 (2010).

11. A. D. Attie and R. T. Raines, Analysis of Receptor-Ligand Interactions, J. Chem. Educ. 72, 119 (1995). 
12. J. K. Kim and J. J. Tyson, Misuse of the Michaelis-Menten Rate Law for Protein Interaction Networks and Its Remedy, PLoS Comput. Biol. 16, e1008258 (2020).

13. S. Schnell and P. K. Maini, A Century of Enzyme Kinetics: Reliability of the $K_{\mathrm{M}}$ and $v_{\max }$ Estimates, Comm. Theor. Biol. 8, 169-187 (2003).

14. L. A. Segel and M. Slemrod, The Quasi-Steady-State Assumption: a Case Study in Perturbation, SIAM Rev. 31, 446-477 (1989).

15. A. Fujioka, K. Terai, R. E. Itoh, K. Aoki, T. Nakamura, S. Kuroda et al., Dynamics of the Ras/ERK MAPK Cascade as Monitored by Fluorescent Probes, J. Biol. Chem. 281, 89178926 (2006).

16. N. Blu"thgen, F. J. Bruggeman, S. Legewie, H. Herzel, H. V. Westerhoff, and B. N. Kholodenko, Effects of Sequestration on Signal Transduction Cascades, FEBS J. 273, 895-906 (2006).

17. S. Carmi, E. Y. Levanon, S. Havlin, and E. Eisenberg, Connectivity and Expression in Protein Networks: Proteins in a Complex Are Uniformly Expressed, Phys. Rev. E. 73, 031909 (2006).

18. J. K. Kim and D. B. Forger, A Mechanism for Robust Circadian Timekeeping via Stoichiometric Balance, Mol. Syst. Biol. 8, 630 (2012).

19. A. M. Bersani, E. Bersani, G. Dell'Acqua, and M. G. Pedersen, New Trends and Perspectives in Nonlinear Intracellular Dynamics: One Century from Michaelis-Menten Paper, Contin. Mech. Thermodyn. 27, 659-684 (2015).

20. N. E. Buchler and M. Louis, Molecular Titration and Ultrasensitivity in Regulatory Networks, J. Mol. Biol. 384, 1106-1119 (2008).

21. A. R. Tzafriri, Michaelis-Menten Kinetics at High Enzyme Concentrations, Bull. Math. Biol. 65, 1111-1129 (2003).

22. H. C. Lim, On Kinetic Behavior at High Enzyme Concentrations, AICHE J. 19, 659-661 (1973).

23. S. Cha, Kinetic Behavior at High Enzyme Concentrations: Magnitude of Errors of Michaelis-Menten and Other Approximations, J. Biol. Chem. 245, 4814-4818 (1970).

24. K. J. Laidler, Theory of the Transient Phase in Kinetics, with Special Reference to Enzyme Systems, Can. J. Chem. 33, 1614-1624 (1955).

25. F. Gachon, E. Nagoshi, S. Brown, J. Ripperger, and U. Schibler, The Mammalian Circadian Timing System: From Gene Expression to Physiology, Chromosoma 113, 103112 (2004).

26. D. H. Nagel and S. A. Kay, Complexity in the Wiring and Regulation of Plant Circadian Networks, Curr. Biol. 22, R648-R657 (2012).

27. T. S. Hatakeyama and K. Kaneko, Reciprocity between Robustness of Period and Plasticity of Phase in Biological Clocks, Phys. Rev. Lett. 115, 218101 (2015).

28. N. Mosheiff, B. M. C. Martins, S. Pearl-Mizrahi, A. Grünberger, S. Helfrich, I. Mihalcescu, D. Kohlheyer, J. C. W. Locke, L. Glass, and N. Q. Balaban, Inheritance of Cell-Cycle Duration in the Presence of Periodic Forcing, Phys. Rev. X 8, 021035 (2018).

29. V. Kampen and N. Godfried, Stochastic Processes in Physics and Chemistry (Elsevier, 1992).

30. E. Levine and T. Hwa, Stochastic Fluctuations in Metabolic Pathways, Proc. Natl. Acad. Sci. USA 104, 9224-9229 (2007).

31. W.-Y. Kim, S. Fujiwara, S.-S. Suh, J. Kim, Y. Kim, L. Han et al., ZEITLUPE is a Circadian Photoreceptor Stabilized by GIGANTEA in Blue Light, Nature 449, 356-360 (2007).

32. J. Y. Cha, J. Kim, T.-S. Kim, Q. Zeng, L. Wang, S. Y. Lee, W.-Y. Kim, and D. E. Somers, GIGANTEA Is a Co-Chaperone Which Facilitates Maturation of ZEITLUPE in the Arabidopsis Circadian Clock, Nat. Commun. 8, 3 (2017).

33. C.-M. Lee, M.-W. Li, A. Feke, W. Liu, A. M. Saffer, and J. M. Gendron, GIGANTEA Recruits the UBP12 and UBP13 Deubiquitylases to Regulate Accumulation of the ZTL Photoreceptor Complex, Nat. Commun. 10, 3750 (2019). 
34. C. Lee, J. P. Etchegaray, F. R. Cagampang, A. S. Loudon, and S. M. Reppert, Posttranslational Mechanisms Regulate the Mammalian Circadian Clock, Cell 107, 855867 (2001).

35. A. C. Liu et al., Redundant Function of REV-ERB $\alpha$ and 8 and Non-Essential Role for Bmal1 Cycling in Transcriptional Regulation of Intracellular Circadian Rhythms, PLoS Genet. 4, e1000023 (2008).

36. N. Preitner et al., The Orphan Nuclear Receptor REV-ERBa Controls Circadian Transcription within the Positive Limb of the Mammalian Circadian Oscillator, Cell 110, 251-260 (2002).

37. E. L. McDearmon et al., Dissecting the Functions of the Mammalian Clock Protein BMAL1 by Tissue-Specific Rescue in Mice, Science 314, 1304-1308 (2006).

38. B. C. Goodwin, Oscillatory Behavior in Enzymatic Control Processes, Adv. Enzyme Regul. 3, 425-438 (1965).

39. R. Lim, J. Chae, D. E. Somers, C.-M. Ghim, and P.-J. Kim, Cost-Effective Circadian Mechanism: Rhythmic Degradation of Circadian Proteins Spontaneously Emerges without Rhythmic Post-Translational Regulation, iScience 24, 102726 (2021).

40. M. Zhou, J. K. Kim, G. W. Eng, D. B. Forger, and D. M. Virshup, A Period2 Phosphoswitch Regulates and Temperature Compensates Circadian Period, Mol. Cell 60, 77-88 (2015).

41. E. M. Farre' and S. A. Kay, PRR7 Protein Levels Are Regulated by Light and the Circadian Clock in Arabidopsis, Plant J. 52, 548-560 (2007).

42. H.-H. Jo, Y. J. Kim, J. K. Kim, M. Foo, D. E. Somers, and P.-J. Kim, Waveforms of Molecular Oscillations Reveal Circadian Timekeeping Mechanisms, Commun. Biol. 1, 207 (2018).

43. G. van Ooijen, L. E. Dixon, C. Troein, and A. J. Millar, Proteasome Function Is Required for Biological Timing throughout the Twenty-Four Hour Cycle, Curr. Biol. 21, 869-875 (2011).

44. J. Chae, R. Lim, C.-M. Ghim, and P.-J. Kim, Backward Simulation for Inferring Hidden Biomolecular Kinetic Profiles, STAR Protoc. 2, 100958 (2021).

45. W. Stroberg and S. Schnell, On the Estimation Errors of $K_{M}$ and $V$ from Time-Course Experiments Using the Michaelis-Menten Equation, Biophys. Chem. 219, 17 (2016).

46. B. Choi, G. A. Rempala, and J. K. Kim, Beyond the Michaelis-Menten Equation: Accurate and Efficient Estimation of Enzyme Kinetic Parameters, Sci. Rep. 7, 1-11 (2017).

47. M. J. D. Powell, An Efficient Method for Finding the Minimum of a Function of Several Variables without Calculating Derivatives, Comput. J. 7, 155-162 (1964).

48. C.-M. Ghim and E. Almaas, Two-Component Genetic Switch as a Synthetic Module with Tunable Stability, Phys. Rev. Lett. 103, 028101 (2009).

49. P.-J. Kim and N. D. Price, Macroscopic Kinetic Effect of Cell-to-Cell Variation in Biochemical Reactions, Phys. Rev. Lett. 104, 148103 (2010).

50. N. Nakamichi et al., PSEUDO-RESPONSE REGULATORS 9, 7, and 5 Are Transcriptional Repressors in the Arabidopsis Circadian Clock, Plant Cell 22, 594-605 (2010). 